\title{
AIRTIME TRANSFERS AND MOBILE COMMUNICATIONS: EVIDENCE IN THE AFTERMATH OF NATURAL DISASTERS*
}

\author{
Joshua Blumenstock, University of Washington \\ Nathan Eagle, Santa Fe Institute \\ Marcel Fafchamps, Oxford University
}

December 31, 2015

\begin{abstract}
We provide empirical evidence that Rwandans use the mobile phone network to transfer airtime to those affected by unexpected shocks. Using an extensive dataset on mobile phone activity in Rwanda and exploiting the quasi-random timing and location of natural disasters, we show that individuals make transfers and calls to people affected by disasters. The magnitude of these transfers is small in absolute terms, but statistically significant; in response to the Lake Kivu earthquake of 2008, we estimate that roughly US\$84 in airtime was transferred to individuals in the affected region, that $70 \%$ of these transfers were immediately used to make outgoing calls, and that US\$16,959 was spent calling those near the epicenter. Unlike other forms of interpersonal transfers, mobile airtime airtime is sent over large geographic distances and in response to covariate shocks. Transfers are more likely to be sent to wealthy individuals, and are sent predominantly between pairs of individuals with a strong history of reciprocal favor exchange.
\end{abstract}

JEL Classification: O17, H84, O16, O33

\footnotetext{
*The authors are grateful for thoughtful comments from Alain de Janvry, Stefano DellaVigna, Frederico Finan, Mauricio Larrain, Ethan Ligon, Jeremy Magruder, Edward Miguel, Alex Rothenberg, Elisabeth Sadoulet, and seminar participants at NEUDC, PACDEV, MWIEDC, and the Berkeley Development Lunch. We gratefully acknowledge financial support from the International Growth Center, the National Science Foundation, the Institute for Money, Technology and Financial Inclusion, and the NET Institute. All errors are our own.
}

(C) 2016. This manuscript version is made available under the Elsevier user license http://www.elsevier.com/open-access/userlicense/1.0/ 


\section{Introduction}

In the aftermath of unexpected economic shocks, people often rely on friends and family for support in cash and in kind. In developing countries, support has historically been limited by weak infrastructure for communicating with and assisting others. As a result, most empirical evidence indicates that assistance and favors are primarily exchanged within small, geographically-defined communities (Udry 1994, Fafchamps \& Gubert 2007, de Weerdt \& Fafchamps 2010).

In recent years, the proliferation of mobile phones and of phone-based financial services has provided billions of individuals in developing countries with a new mechanism for communication and interpersonal transfers. With roughly 250 deployments in the global south, such "branchless banking" systems allow individuals to transfer money from one phone to another at a fraction of the cost of existing alternatives (McKay \& Pickens 2010, GSMA 2014). In Kenya, for instance, where over US\$200 million is transferred over the system per day, individuals with access to the mobile money network are better able to smooth consumption than those without (Suri et al. 2012, Jack \& Suri 2014, Pulver 2009).

We exploit a novel source of data on mobile phone use to better understand how individuals use the mobile phone network to cope with unexpected shocks. We observe the entire universe of mobile phonebased communications in Rwanda from 2005 through 2009, including transaction logs that contain detailed information on millions of interpersonal transfers of mobile airtime and billions of phone calls. Our primary results are identified by a magnitude 6.0 earthquake in the Lake Kivu region of Rwanda, which left 43 dead and 1,090 injured, and caused significant disruption in public utilities and other local infrastructure. While Rwanda did not have a functioning mobile money system at the time of this earthquake, we observe that in the earthquake's immediate aftermath, individuals living across Rwanda transferred airtime and initiated phone calls to individuals living close to the epicenter. We estimate that an additional US\$84 in airtime was transferred, and that US\$16,959 was spent making phone calls, to affected individuals. The economic significance of the airtime transfers was thus modest, though this is due in part to the fact that only 1,400 individuals in the region had ever used the airtime transfer service prior to the earthquake, creating a small population of potential recipients. The value of the phone calls was much higher, though more difficult to interpret. Since in Rwanda the caller bears the full cost of making a phone call, this may represent an implicit transfer of communication costs, but more likely it simply expresses the desire of the calling party to communicate in a time of crisis. Our empirical results are robust to the inclusion of dyad fixed effects, time dummies, and time-varying controls. We further provide several robustness checks and placebo tests to show that the results are not simply driven by the large number of observations in our dataset.

Our analysis reveals significant heterogeneity in the nature of transfers sent in response to unexpected 
shocks. Using several different proxies for socioeconomic status based on follow-up interviews with a representative sample of mobile phone subscribers, we find that while wealthier individuals are more likely to recieve transfers under normal circumstances, they are even more likely to receive transfers after natural disasters. The recipients of shock-induced transfers also have larger social networks, and are more centrally positioned within their network. Along several additional dimensions, it is the historically privileged strata of Rwandan society who appear to benefit most from access to mobile phone-based transfers.

Finally, we analyze the pattern of interpersonal transfers to shed light on the motives that cause people to make transfers to those impacted by covariate shocks. Our goal is to test whether the observed transfers are more consistent with a model of giving based on pure charity (Becker 1974, Andreoni \& Miller 2002) or with a model of conditional reciprocity where individuals give because they wish to receive in the future (e.g., Ligon et al. 2002, Foster \& Rosenzweig 2001, Falk \& Fischbacher 2006). While our ability to cleanly differentiate between these models is limited by the observational data at our disposal, three stylized facts in our data appear broadly consistent with a model based on reciprocity. First, there is a strong history-dependence of transfers sent in response to large shocks, in that after the earthquake people are more likely to send funds to people from whom they have received in the past. Second, it is the wealthier individuals who receive the largest volume of transfers in the immediate aftermath of the earthquake, not the poorer individuals that one would expect in a naive model of charity. Third, post-quake transfers decrease with the geographic distance between individuals, even when controlling for social distance and unobserved, time-invariant dyadic heterogeneity.

To summarize, the evidence indicates that Rwandans use airtime transfers to help each other cope with large economic shocks, that benefits from these transfers are not uniformly distributed, and that the pattern of transfers is consistent with conditional reciprocity. To the extent that airtime transfers can be interpreted as a form of favor exchange, there are two features that distinguish the mobile phone-based response from much of the evidence on favor exchange, especially traditional risk sharing. First, whereas traditional risk sharing networks are constrained by geography (Udry 1994, Fafchamps \& Gubert 2007), transfers sent over the mobile phone network are sent over large distances, often in excess of $100 \mathrm{~km}$. Second, these transfers are sent in response to large, covariate shocks, rather than the geographically isolated, idiosyncratic shocks that are the focus of much of the existing risk sharing literature (Townsend 1994, De Vreyer et al. 2010, Gine \& Yang 2009).

These findings complement recent work by Jack \& Suri (2014), who use consumption data to show that Kenyans with access to mobile money are better able to smooth consumption than those without. It also relates closely to a growing body of research concerned with understanding the economic impact of mobile phones and other information and communication technologies (ICTs) in developing economies. Recent work 
in this area describes how mobile phones can, under certain conditions, reduce information asymmetries and search costs in agricultural markets (Jensen 2007, Aker 2008, Fafchamps \& Minten 2012, Aker \& Fafchamps 2014), lower transaction costs (Jack \& Suri 2014), and potentially provide an alternative device for savings (Mbiti \& Weil 2011, Mas \& Mayer 2011, Blumenstock et al. 2015).

We make two methodological contributions that we hope can help facilitate the use of large-scale, networkbased datasets in applied microeconomic and development research. First, we develop an approach to inferring the relative wealth of mobile phone subscribers from their history of mobile phone use. The sole purpose of this approach is to proxy for the relative wealth of transfer recipients at a particular point in time. Second, we develop an algorithm for locational inference that allows us to continuously impute the location of an individual based on her intermittent sequence of phone calls. We use this method to determine whether or not a subscriber is affected by a regional disaster, but the algorithm could be implemented more broadly to identify individuals in need of help.

\section{Background and Context}

The context for our study is Rwanda, a small, landlocked nation of 10 million people, where roughly $90 \%$ of the population survives on subsistence agriculture. We focus our analysis on the period from 2005-2009, a period during which mobile phone penetration increased from roughly 2.5 percent to 33.4 percent - a compound annual growth rate of roughly 74 percent. Such rapid growth has been common in many subSaharan African nations, where landlines are rare and the cost of owning a mobile phone is falling quickly. The cost of mobile telephony during this period represented a significant portion of household expenditures (Ureta 2005). At the time of our study in Rwanda, it cost roughly $\$ 50$ for the phone, and an additional $\$ 0.20$ per minute and $\$ 0.10$ per SMS (Republic of Rwanda 2010, Donner 2008). The ITU estimates the monthly "price basket" for mobile service was $\$ 12.30$ per month, or $\$ 147.60$ per year, which represented a significant portion of the PPP-adjusted GDP per capita of $\$ 800 .^{1}$

Our empirical analysis focuses on interpersonal transfers of airtime funds between mobile subscribers. These transfers were made possible by a rudimentary precursor to "mobile money" that was launched in Rwanda in late 2006 by the monopoly mobile phone operator at the time. In Rwanda, where nearly all phone usage is prepaid and all incoming calls are free, the system works much like a debit card. To make a transfer, individuals buy airtime scratch-cards, ranging from US $\$ 0.10$ to US $\$ 20$, from thousands of stores and street vendors throughout the country. The purchased balance is deposited on the prepaid mobile phone

\footnotetext{
${ }^{1}$ The price basket is based on the prepaid price for 25 calls per month spread over the same mobile network, other mobile networks, and mobile to fixed calls and during peak, off-peak, and weekend times. The basket also includes 30 text messages per month (http://devdata.worldbank.org/ict/rwa_ict.pdf).
} 
account, and can then be used to make phone calls, to send text messages, or to use other mobile services. Using the transfer service, one subscriber can transfer an arbitrary balance, instantaneously and free of charge, to another subscriber. The transferred balance can then be used by the recipient to make calls, or re-transferred to another subscriber or vendor. During the period of time we analyze (2005-2009), there was no formal "cash-out" policy that guaranteed that the recipient could resell airtime to a vendor, but informal cashing-out was commonplace, with vendors typically charging $10-20 \%$ for the transaction. ${ }^{2}$ In 2010 , the phone company launched a fully fledged mobile money service that, among other features, made it possible to cash out airtime at fixed tariffs.

In the past several years, similar systems have become popular in developing countries, where over 1.7 billion people own a mobile phone but do not have a bank account (CGAP and GSMA 2009). The airtime transfer service we focus on was a common antecedent to most modern mobile money deployments (Aker \& Mbiti 2010). Compared to alternative mechanisms for sending money available to Rwandans, sending airtime was considerably cheaper, faster, and more convenient (Table 1). Other options included MoneyGram, Western Union, or the Post Office, but transaction costs across these services ranged from $10-100 \%$ and, at the time of our study, none of these services permitted a transfer of less than US\$10. Rwandans could also send money through a bus or taxi driver, but these transfers were contingent on bus schedules and road conditions - and much slower than airtime transfer.

\section{Identification and Estimation Strategy}

\subsection{The giving response to unexpected shocks}

The focus of our empirical analysis is on airtime transfers sent in response to unpredictable but publicly observed shocks such as earthquakes and floods. We also quantify the volume and value of calls made in response to such shocks. Empirically, we use spatial and temporal variation to identify individuals who are affected by these shocks, then measure how airtime transfers and communication events respond to these unexpected events. The response can be measured at three levels. We start at the regional level, to measure the total response to the shock. We then disaggregate transfers to the level of the individual sender and receiver, to study heterogeneity in who sends and receives. Finally, we disaggregate the analysis to the level of the dyad, which permits us to analyze the types of relationships that are likely to be involved in transfers, and to informally test between competing models of giving.

\footnotetext{
${ }^{2}$ Based on conversations with Rwandan informants at the time. In a 2009 survey of 910 Rwandans, respondents were asked to list "the preferred method for sending money to a friend or family member." 28 percent listed "Airtime Transfer" as their preferred method (Blumenstock \& Eagle 2012). This suggests that, prior to 2010, mobile airtime was perceived as relatively liquid.
} 
Formally, let $\tau_{i j r t}$ denote the gross transfer of airtime (or the total value of the call) sent to an individual $i$, located in region $r$ at time $t$, from another individual $j$. Further define $\tau_{i r t}=\sum_{j} \tau_{i j r t}$ the total gross transfers received by user $i$ in region $r$ at time $t$, and define $\tau_{r t}=\sum_{i} \tau_{i r t}$ as the total gross transfers received by users in location $r$ at time $t$. When analyzing calls instead of airtime transfers, $\tau_{i j r t}$ is the value of airtime spent by $j$ in communicating with $i$. We estimate models of the form:

$$
\begin{aligned}
\tau_{r t} & =\alpha_{1}+\gamma_{1} \text { Shock }_{r t}+\theta_{t}+\pi_{r}+\varepsilon_{r t} \\
\tau_{i r t} & =\alpha_{2}+\gamma_{2} \text { Shock }_{i r t}+\phi N e a r \text { Epicenter }_{i t}+\theta_{t}+\pi_{i}+\varepsilon_{i r t} \\
\tau_{i j r t} & =\alpha_{3}+\gamma_{3} \text { Shock }_{i r t}+\phi N e a r E \text { Eicenter }_{i t}+\theta_{t}+\pi_{i j}+\varepsilon_{i j r t}
\end{aligned}
$$

where $S h o c k_{r t}$ is a dummy variable equal to 1 if location $r$ received a shock at time $t$, and Shock $k_{i r t}$ equals 1 if $t$ is the day of a shock and $i$ was in an affected region $r$ at that time. NearEpicenter ${ }_{i t}$ takes the value 1 at all times when $i$ is near the epicenter of the shock (irrespective of a shock occurring), and controls for the possibility that individuals might receive transfers when visiting the shock-prone regions. Time dummies $\theta_{t}$ control for long-term growth in traffic, as well as day-of-the-week (e.g., week-end) and day-of-the-month (e.g., payday) effects that affect all regions similarly. Location and recipient fixed effects $\pi_{r}$ and $\pi_{i}$ control for the fact that different regions and individuals are more likely to receive transfers on average. Dyadic fixed effects $\pi_{i j}$ control for the average intensity and direction of transfer flows between two individuals. Finally, to minimize the likelihood that our results are driven by differential growth in mobile usage across locations, we restrict the analysis to a specific time window $T_{\min } \leq t_{s} \leq T_{\max }$ around the time of the shock $t_{s}$.

Identification is achieved as in a difference-in-difference framework: parameters $\gamma_{1}, \gamma_{2}$ and $\gamma_{3}$ represent the average treatment effect of the shock on people with access to the mobile network. The exogeneity of $S_{h o c k}$ is guaranteed if its timing could not have been predicted, i.e., the shock constitutes a natural experiment. If $\gamma_{1}>0, \gamma_{2}>0$ and $\gamma_{3}>0$, this is interpreted as evidence that the shock Shock $k_{r t}$ caused an increase in airtime transfers to users in the affected region. We check the robustness of our results in various ways, notably by varying the time window over which the models are estimated, by controlling for several factors that depend on both time and location, and by running a number of falsification and placebo tests. Following Bertrand et al. (2004), in individual and dyadic regressions standard errors are clustered by location (i.e., by the location of the nearest cellular tower). 


\subsection{Heterogeneity in transfers}

To better understand the nature of the response to shocks, we allow for heterogeneous effects for different types of individuals and for different types of dyadic sender-recipient pairs. We denote these individual and dyadic characteristics by $Z_{i}$ and $Z_{i j}$, respectively. As discussed in greater detail in the next section, relevant $Z_{i}$ include $i$ 's wealth and social connectedness, and $Z_{i j}$ include the geographic distance between $i$ and $j$, and information about the history of transfers between $i$ and $j$. Heterogeneity is then esimated using models of the form:

$$
\begin{gathered}
\tau_{i t}=\alpha_{2}+\quad \gamma_{2} \text { Shock }_{i t}+\beta_{2} Z_{i} \text { Shock }_{i t}+\phi_{2} \text { NearEpicenter }_{i t}+ \\
\eta_{2} Z_{i} \text { DayOfShock }_{t}+\zeta_{2} Z_{i} \text { NearEpicenter }_{i t}+\theta_{t}+\pi_{i}+\varepsilon_{i t} \\
\tau_{i j t}=\alpha_{3}+\quad \gamma_{3} \text { Shock }_{i t}+\beta_{3} Z_{i j} \text { Shock }_{i t}+\phi_{3} \text { NearEpicenter }_{i t}+ \\
\eta_{3} Z_{i j} \text { DayOfShock }_{t}+\zeta_{3} Z_{i j} \text { NearEpicenter }_{i t}+\theta_{t}+\pi_{i j}+\varepsilon_{i j t}
\end{gathered}
$$

As before, $\tau_{i j r t}$ denotes the gross transfer of airtime received by an individual $i$, located in region $r$ at time $t$, from another individual $j$, with $\tau_{i r t}=\sum_{j} \tau_{i j r t}$. Shock $k_{i t}$ takes the value 1 if $i$ is affected by the shock and zero otherwise, and is the product of DayOfShock ${ }_{t}$, a dummy variable taking the value one on the day of a severe shock and NearEpicenter ${ }_{i t}$, a dummy variable indicating whether $i$ was close to the shock on day $t$. Double interaction terms of the form $Z_{i}$ DayOfShock $k_{t}$ are included to control for the possibility that, in the country as a whole, variation in $Z_{i}$ affects transfers on the day of the shock differently from other days. Our primary interest is in the estimates of $\beta_{2}$ and $\beta_{3}$, which indicate the types of individuals and dyads that are most likely to receive transfers caused by economic shocks.

\section{Data and Measurement}

The main dataset we use comes from Rwanda's primary telecommunications operator, which held a near monopoly on mobile telephony until $2009 .^{3}$ The data contain a comprehensive log of all activity that occurred on this network between early 2005 and early 2009. In total, we observe detailed information on over 50 billion transactions (including calls, text messages, and airtime transfers and purchases), covering roughly 1 million subscribers over four years. Summary statistics of this dataset are provided in Table 2.

Our empirical analysis focuses on interpersonal transfers of airtime credit sent between one mobile subscriber and another. In our dataset, we observe detailed information on roughly 10 million such transfers. As

\footnotetext{
${ }^{3}$ During the window of time we examine, the operator we focus on maintained over $90 \%$ market share of the mobile market. The company's primary competitor did not gain traction in the market until the end of 2008, and only in 2010 did the market become competitive. The number of landlines in Rwanda is negligible (roughly $0.25 \%$ penetration).
} 
shown in Figure 3, the system was not heavily utilized prior to 2008, when the operator launched a nationwide promotion and uptake dramatically increased. We focus on transactions sent prior to this growth stage. For each transaction, we observe the date, time, and value of the transfer, as well as a unique (anonymized) identifier for both the sender and recipient. To estimate the regressions described in sections 3 , we aggregate this raw data on each day for each region (equation 1), individual (equation 2), and dyadic pair of individuals (equation 3). This allows us to measure, at three different levels of aggregation, the net and gross volume of airtime received on each day.

\subsection{Physical Location}

The identification strategy we employ relies on spatial, as well as temporal, variation in transfers. Therefore, it is important that we be able to assign each individual, on each day, to an approximate geographic location. In standard GSM cellular networks, such as the one in place in Rwanda, the phone company does not record continuous GPS coordinates of each subscriber. However, each phone call in the database is tagged with an originating and terminating tower, which allows us to roughly place the caller and recipient at the time of the call.

We use these discrete events to approximate the continuous trajectory of each user through time and space, based on the intermittent sequence of phone calls logged by the mobile operator. As can be seen in Figure 1, which shows the spatial distribution of cell phone towers in early 2008, towers in rural areas are relatively sparse, with a median distance between towers of $68 \mathrm{~km}$. The locational inference algorithm employs a smoothing function $K(\cdot)$ to predict the unknown location of $i$ at time $t$ from the kernel-weighted Euclidean centroid of $i$ 's known locations at times in the vicinity of $t$ (see Figure 2).

Formally, we estimate the unknown location $\widehat{r_{i t}}$ of individual $i$ at time $t$ as

$$
\widehat{r_{i t}}=\frac{1}{N_{i t}} \sum_{s=T_{\min }}^{T_{\max }} K\left(\frac{t-s}{h}\right) \cdot \widehat{q_{i s}}
$$

where $N_{i t}$ is the total number of phone calls made by $i$ within a window of time $\left[T_{\min }, T_{\max }\right]$ around $t$, and $\widehat{q_{i s}}$ is the (known) location of the tower used at time $s$. The kernel $K(x)$ is a symmetric function that integrates to one, which specifies the extent to which additional weight is placed on calls close in time to $t$. In our results we use a uniform kernel such that $K(u)=1 / N_{i}$, however very little changes if a different kernel is specified. 


\subsection{Characteristics of individuals and dyads}

To estimate our heterogeneous effects models, we require characteristics of individuals $\left(Z_{i}\right)$ and dyads $\left(Z_{i j}\right)$. Many of these can be computed directly from the anonymized call records dataset, including the following:

- Physical distance between $i$ and $j\left(D_{i j t}\right)$ : To estimate the distance between $i$ and $j$ at time $t$, we first compute the (latitude,longitude) locations $\widehat{r_{i t}}=\left(\phi_{i}, \lambda_{i}\right)$ and $\widehat{r_{j t}}=\left(\phi_{j}, \lambda_{j}\right)$ using the locational inference algorithm described in Section 4.1. Then, we compute the arc distance $D_{i j t}$ using the haversine formula:

$$
D_{i j t}=2 r \cdot \arcsin \left(\sqrt{\sin ^{2}\left(\frac{\Delta \phi}{2}\right)+\cos \phi_{i} \cos \phi_{j} \sin ^{2}\left(\frac{\Delta \lambda}{2}\right)}\right)
$$

where $\Delta \phi=\left|\phi_{i}-\phi_{j}\right|, \Delta \lambda=\left|\lambda_{i}-\lambda_{j}\right|$ and $r=6356.78$ is the radius of the earth.

- Social connectedness $\left(S_{i j}\right)$ : To measure the strength of the social connection between $i$ and $j$, we calculate $S_{i j}$ in two ways: first, following Marmaros \& Sacerdote (2006) we count the total number of non-monetary interactions (phone calls and text messages) between $i$ and $j$ in a 3-month window. Second, as in Karlan et al. (2009), we measure the maximum network flow as the number of distinct paths between $i$ and $j$ in the complete undirected call graph.

- Past transfer history $\left(T_{i j t}\right)$ : Since we observe all transactions between $i$ and $j$ over a 4 -year period, it is possible at any point $t$ to compute the gross balance of payment sent from $j$ to $i$ as $T_{i j t}=\sum_{s<t} \tau_{i j s}$ and from $i$ to $j$ as $T_{j i t}=\sum_{s<t} \tau_{j i s}$. The net balance of transfers $T_{i j t}^{n e t}$ is simply $T_{i j t}-T_{j i t}$.

- Individual Characteristics $\left(Z_{i}\right)$ : In addition, we compute a large number of characteristics that are summarized for the survey sample in Appendix Table 7, including:

- Total expenditures on airtime: The amount spent on making calls and sending text messages.

- Days of activity: The number of different days on which the phone was used.

- Call activity: Number of outgoing and incoming calls. We separately looking at outgoing and incoming calls, outgoing and incoming text messages, and international call activity.

- Degree: Number of unique contacts with whom the person communicates.

- Clustering: Percentage of first-degree contacts that have contacted each other.

- Betweenness: Average shortest path between the user and 50 randomly sampled numbers.

\subsection{Inferred socioeconomic status}

In assessing heterogeneity in mobile phone-based activity, we are interested in understanding the extent to which wealthier or poorer individuals are more likely to receive interpersonal transfers following economic shocks. However, since our data are anonymized and contain no demographic or economic characteristics, we 
do not directly observe the socioeconomic status (SES) of individual subscribers. Yet, wealthy individuals tend to have different communication patterns than poorer individuals, and there is good reason to suspect that mobile phone use is highly correlated with SES (Blumenstock \& Eagle 2012). For instance, we observe that wealthy people tend to make a larger number of international calls, poorer individuals tend to receive more calls than they make (because only the caller pays for the cost of the call in Rwanda), and poorer individuals tend to buy airtime in smaller denominations. Thus, we construct a composite indicator of SES that indicates the predicted wealth of each mobile phone subscriber, where the prediction is based on the subscriber's anonymous history of calls. We briefly summarize this approach here, and discuss in much greater detail the limitations of this approach, as well as a number of robustness checks that we run to test the validity of this method, in Section (iv).

1. Rwandan Household Survey (RHS): We use a nationally representative household survey conducted by the Rwandan government in 2005 on a representative set of 6,900 households. The survey contains roughly five hundred questions typical of Living Standard and Measurement Surveys, and includes a detailed module on demographic composition and socioeconomic status (National Institute of StatisticsRwanda 2007). Most relevant to the current analysis, roughly seventy questions were asked about asset ownership and household expenditures, which makes it possible to estimate each household's annual expenditures in a manner following Deaton \& Zaidi (2002).

2. Phone survey conducted by authors: In 2009, we conducted a phone survey of a geographically stratified group of Rwandan mobile phone users. Using a trained group of enumerators from the Kigali Institute of Science and Technology (KIST), a short, structured interview was administered to roughly 1,000 individuals. In the phone survey, we collected answers to a small subset of the RHS questions (described above) about household asset ownership and housing characteristics. The survey instrument contained a maximum of 80 questions and took between 10 and 20 minutes to administer. Complete details on the administration of this phone survey are provided in Blumenstock \& Eagle (2010). ${ }^{4}$

\footnotetext{
${ }^{4}$ Most relevant to this paper, the survey population was designed to be representative sample of active phone users. Starting with a population of 800,000 active subscribers (in 2009), each individual was assigned to a district based on the location from which the majority of his or her calls were made. We then randomly sampled 300 numbers from each of 30 districts, creating a base survey population of 9,000 candidate respondents. Our survey team called roughly 10 percent of this candidate population. Conditional on the responding picking up the phone, response rates were extremely high (98\%); however, roughly $38 \%$ of respondents never picked up the phone, even after three attempts were made. To correct for non-response, sampling weights for each district were determined based on the distribution of districts in the set of 800,000 active numbers, and survey weights are assigned to make the proportion of respondents equal to the proportion of active phone users in each district.

However, as discussed in greater detail in Blumenstock \& Eagle (2010), there are only modest differences between the group of individuals who participated in the phone survey and those who did not. There is a large and significant difference in the number of days during which the phone is used (likely driven by the fact that many of the non-respondents have switched SIM card), but there are not statistically signficant differences in the level of activity per day in which the phone is active (including calls per day, SMS per day, degree centrality, total airtime purchases, etc.).
} 
Utilizing these data, we follow a 3-step process to model the relationship between phone use and wealth, wherein we (i) use the Rwandan RHS data to model the relationship between annualized expenditures and assets; (ii) use the asset-based questions from the phone survey to create a measure of predicted annualized expenditures for this sample; and (iii) merge the call records from this sample with the predicted annualized expenditures to model the relationship between SES and phone use. These steps are explained in greater detail below.

\section{(i) Modeling the relationship between assets and expenditures:}

Using a nationally-represenative Rwandan Household Survey (RHS) that contains detailed consumption and expenditure information (National Institute of Statistics-Rwanda 2007), we first estimate a hedonic regression of annual expenditures $Y_{i d}$ of household $i$ in district $d$ on fixed assets $A_{i d}$ and housing characteristics $H_{i d}$.

$$
Y_{i d}=\alpha+\sum_{j}^{h_{\max }} \beta_{j} H_{i d}+\sum_{k}^{a_{\max }} \delta_{k} A_{i d}+\mu_{d}+\epsilon_{i d}
$$

The quantities $Y_{i d}, A_{i d}$, and $H_{i d}$ are all captured in the RHS, and $\mu_{d}$ is a district fixed effect. Equation (6) predicts the annual expenditure $\widehat{Y}_{i d}$ of a household on the basis of observable $A_{i d}$ and $H_{i d}$. This is conceptually similar to a proxy-means test (cf. Montgomery et al. 2000), wherein we regard $\widehat{Y}_{i d}$ is as a proxy for permanent income. Appendix Table 6 gives the coefficients from the linear terms that result from estimating (6). It is evident that annual expenditures are heavily correlated with asset ownership. ${ }^{5}$

\section{(ii) Predicting the expenditures of phone survey respondents:}

After estimating (6) on the RHS data, we obtain a vector of coefficients $\widehat{\beta}_{j}$ and $\widehat{\delta_{k}}$ that can be used to predict total expenditures given knowledge of assets and housing characteristics $A_{i d}$ and $H_{i d}$. Thus, for any individual in Rwanda, we could in principle predict that individual's annual expenditures, denoted by $\widehat{Y_{i d}}$, by asking that individual a small number of questions about his household. In our case, we conducted the phone survey described above in which, for each surveyed individual, we collected a limited set of $H_{i d}$ and $A_{i d}$ through a short, phone-based instrument. The questions included in this survey were those where we observed a significant unconditional correlation with annualized expenditures in the RHS data, and where our piloting indicated that a reliable response could be obtained over the phone. ${ }^{6}$ Using the $H_{i d}$ and $A_{i d}$

\footnotetext{
${ }^{5}$ To reduce the potential bias of outliers, we remove outliers with abnormally large studentized residuals, following a standard process described in (Fox 1997). However, our results change very little if we use an alternate technique for removing outliers, such as removing the top $1 \%$ or $5 \%$ of extreme values.

${ }^{6}$ We found that several questions which were highly predictive of annual expenditures in the RHS data were not well-suited to a phone-based survey. For instance, in pilot surveys our ennumerators found that consumption and expenditure modules were time consuming and reported low confidence in respondents' answers. Other questions, such as the amount of land owned by the respondent, yielded noisy responses with inconsistent units of measurement, and seemed likely to yield non-traditional
} 
collected through the phone survey, we than calculate the predicted annual expenditures $\widehat{Y}_{i d}$ for each phone survey respondent using the coefficient estimates from equation (6).

\section{(iii) Predicting predicted expenditures of full population of mobile phone subscribers:}

Using the above technique, it is possible to obtain the predicted expenditures $\widehat{Y_{i d}}$ for each of the individuals contacted in the phone survey. We then compute, for each phone user, a vector of phone usage variables $X_{i r}$ thought to be correlated with income, such as the total number of calls made and the average amount of airtime purchased over a given time interval. A subset of these variables are reported for the sample of phone survey respondents in Appendix Table 7. We then fit a flexible model of the form:

$$
\widehat{Y}_{i d}=f\left(X_{i r}\right)
$$

and estimate $f($.$) using data from the phone user survey. In estimating equation (7) we exclude all data on$ interpersonal transfers from $X_{i r}$ to help reduce potential endogeneity when estimating heterogeneous effects models (4) and (5), where $\tau_{i j t}$ is the dependent variable. Instead, $X_{i r}$ consists primarily of characterstics of each subscriber's history of phone calls, text messages, airtime purchases, and social network structure. Appendix Table 8 presents the results from regressing predicted expenditures on several metrics of phone use, polynomial (squared) terms, and district fixed effects.

In the heterogeneous effects specifications that rely on a measure of individual SES, we use the predicted

$\widehat{\widehat{Y}}_{i d}$ obtained by applying the estimated flexible function $\widehat{f}($.) to the full sample of 1.5 million phone users. This particular metric is not intended to be externally valid to the at-large population, for indeed there are significant differences in the wealth distribution of mobile phone owners and non-owners (Blumenstock \& Eagle 2010). Instead, it is intended to estimate whether the mobile phone owners receiving transfers are wealthy or poor relative to other mobile phone owners. We discuss alternate measures of SES in Section (i), and concerns about the potential endogeneity of these measures in Section 6.2 (iv).

\section{Results}

Our identification strategy requires a shock $\left(S h o c k_{r t}\right)$ that is exogenous to mobile phone activity. The primary shock that we exploit is a large earthquake that occurred in the Western Rusizi and Nyamasheke districts of Rwanda on February 3, 2008. The magnitude 6 earthquake left 43 dead and 1,090 injured. It destroyed 2,288 houses and caused regional school closures and electrical outages (though only one cell tower

measurement error. As calls to respondents were billed per second, and given limited survey resources, we sought a balance between predictive power and perceived reliability. 
of 267 was affected). The effects of the earthquake, though large, were geographically contained. The United States Geographical Survey estimates an impacted radius of approximately 20 kilometers from the epicenter - see Figure 1. Based on news reports and discussions with individuals in Rwanda, it does not appear that any particular demographic subgroup of the population was disproportionately affected by this earthquake, and in particular, rich and poor households appear to have been similarly affected (USGS 2009). ${ }^{7}$ This event is ideal for our estimation strategy since the shock is both unpredictable and precisely located in time and space. In Appendix B we show that our results are robust to using alternative measures of Shock $k_{r t}$.

We begin by estimating models (1)-(3) to measure the causal impact of the earthquake on interpersonal transfers and phone calls. We then turn to models (4) and (5) to better understand heterogeneity in the observed pattern of transfers. A full discussion of the robustness of the results, as well as possible alternative explanations, is deffered until Section 6.2, after all results have been presented.

\subsection{The earthquake's impact on transfers and calls}

\section{(i) Baseline results}

To measure the effect of the earthquake on transfers received by impact individuals, we estimate equation (1) at the regional level, and present the results in Panel A of Table 3. The dependent variable $\tau_{r t}$ is the total value of transfers received on day $t$ by region $r$. Corresponding estimates of the earthquake's impact on the value of calls made to affected individuals are presented in Panel B. Our regressions use data from 30 days before to 30 days after the earthquake, though we later demonstrate that our results change little if we use a different time window. Region and day fixed effects are included to control for systematic differences across districts and over time. The variable Shock $k_{r t}$ equals one on February 3rd 2008, the day of the earthquake, in regions affected by the earthquake. ${ }^{8}$

Column (1) defines $r$ at the level of the political district (of which there are 30); column (2) defines $r$ at the level of the cell tower (of which there are 267). The latter specification is advantageous as each geographic unit is smaller and therefore allows us to more precisely identify the regions affected by the quake. To provide context for the later heterogeneous effects models, columns (3) and (4) repeat the estimation at the level of the individual user and of the dyadic pair of individuals. In column (3), the dependent variable $\tau_{\text {irt }}$ is the amount of airtime transferred to individual $i$ in location $r$ at time $t$; in column (4), it is the amount transferred to $i$ from $j$. Individuals who never receive airtime transfers are excluded from column (3) since

\footnotetext{
${ }^{7}$ Much of the damage was sustained in and around the town of Cyangugu, where a relatively representative subset of the population resides. See, for instance, http://earthquake.usgs.gov/eqcenter/eqinthenews/2008/us2008mzam/

${ }^{8}$ For the district-level specification, affected regions are the districts of Rusizi and Nyamasheke. For the tower-level specification, it is towers within $20 \mathrm{~km}$ of the epicenter, though similar point estimates and standard errors are produced if we redefine affected areas as those lying anywhere between 10 to 50 miles of the epicenter.
} 
they do not help identify the effect of the shock (this leaves roughly 110,000 unique individuals); pairs in which $i$ never receives airtime from $j$ are similarly excluded from column (4), leaving roughly 180,000 valid dyads.

We are primarily interested in the coefficient on "Earthquake Shock," which indicates the extent to which an anomalous volume of mobile airtime was sent to individuals in regions affected by the earthquake. While the magnitude of the coefficient depends on the level of aggregation, we find that the effect is statistically significant across all regressions, with T-statistics between 7 and 16. The effect is small in magnitude, however. For instance, we estimate that the earthquake caused an additional US\$84.34 (42,169 RWF) to be sent to each district affected by the earthquake, and an additional US\$0.02 (8 RWF) to be sent between each $i$ - $j$ pair, where $j$ lives close to the earthquake's epicenter. We discuss the economic significance of these point estimates, as well as those from the other panels of Table 3, in greater detail in Section 6 .

\section{$5.2 \quad$ Heterogeneity}

Having observed the direct impact of the earthquake on transfers sent to the affected region, we now turn to the heterogeneity in transfers received and sent. We are primarily concered with understanding the type of person involved in these transfers, the geographic extent of transfers, and whether the past history of transfers between individuals is correlated with shock-induced transfers. As before, we include data from 30 days before to 30 days after the earthquake, and compute $Z_{i j}$ using data from 2007. In all specifications we include a vector of daily fixed effects and interactions between $Z_{i j}$ and DayOfQuake $e_{t}$ and NearEpicenter Nit $_{\text {, }}$ though these coefficients are omitted from most tables for clarity of presentation. Interacted regressors $Z_{i j}$ have been standardized so that all estimated coefficients can be interpreted as the effect of a one standard deviation change on the dependent variable.

In our preferred specification (column 3 of Tables $4-7$ ), we additionally include individual and pairwise fixed effects $\pi_{i j}$ to reduce potential bias from time-invariant omitted variables. However, inclusion of these fixed effects makes it impossible to estimate the unconditional effect of $Z_{i j}$ on $\tau_{i j}$, i.e., whether certain characteristics make transfers more likely on non-shock days. Thus, we follow Wooldridge (2005) and separately recover the average partial effects by obtaining the predicted $\widehat{\tau_{i j}}$ from (5) and then regressing these predicted values on $Z_{i j}$ (column 2). We also include specifications with no fixed effects as a point of reference (column 1), though as noted these estimates are likely to be biased. In all specifications, we include a full set of interactions between $S_{i j}$ and $S h o c k_{i t}$, in order to reduce the potential bias that other factors correlated with $Z_{i j}$ (such as how much $i$ and $j$ like one another, or whether they are related) are spuriously driving the effect we attribute to $Z_{i j}$. The measure $S_{i j}$ used in our main specifications is simply the total number of phone 
calls observed between $i$ and $j$ in the year prior to $t$; Appendix B shows that our results are also robust to a different measure of $S_{i j}$ - the number of shared contacts between $i$ and $j$ - proposed by Karlan et al. (2009).

\section{(i) Wealth}

To measure the marginal effect of the wealth of the sender and recipient on transfers, we use as a wealth proxy the predicted expenditure variable $\widehat{\widehat{Y}}_{i d}$ described in Section 4. To avoid the possibility that results are driven by differences between high- and low-usage individuals (i.e. that richer users may receive more airtime but also transfer more to others), we use net transfers as the dependent variable, though we find similar results with respect to gross transfers. Results are presented in Table 4 .

The primary coefficient of interest is the interaction between the wealth of the recipient $x_{i}$ and the Shock $k_{i r t}$ dummy. The estimates in the second row of Table 4 indicate that wealthier individuals are more likely to receive transfers in the immediate aftermath of the earthquake. This effect exists conditional on the wealth of the sender $x_{j}$, and on the normal level of transfers between $i$ and $j$, as captured in the dyad-specific fixed effects. The former control is important because it limits the possibility that wealthier individuals are receiving more simply because they have wealthier friends, and not because of their own wealth. The latter rules out the possibility that the effect is caused by time-invariant aspects of the $i$ - $j$ relationship, for instance that wealthy $i$ 's may always receive more from $j$, even in transactions that are unrelated to economic shocks. ${ }^{9}$ Note that when using our predicted measure of wealth to estimate models (4) and (5), we must account for the fact that our interaction term $Z_{i}$ is a generated regressor (Murphy \& Topel 2002). Thus, in addition to reporting standard errors in parenthesis (generated from traditional estimation), Table 4 reports p-values obtained from bootstrapping the full series of wealth-related estimations - i.e., the sequence of models (6), (7), and (5) - over 1,000 iterations. ${ }^{10}$

While our preferred proxy for wealth is the measure of predicted expenditures described in section 4.3 , we note that qualitatively similar results obtain for a variety of reasonable alternative indicators of individual SES. For instance, Column 3 of Table 5 reports results from estimating (5) where the total number of airtime

\footnotetext{
${ }^{9}$ In the final three rows of the table, it is evident that, on days without shocks, wealthy individuals are more likely to both send and receive airtime, and that dyads with strong social ties are more likely send money. While these results inform our understanding of general patterns of transfers, our primary focus is on transfers identified by shocks, which inform our interpretation of these transfers as a form of risk sharing.

${ }^{10}$ For each iteration, we draw a random sample (S1) with replacement from the RHS data (6,900 original observations). This sample is used to fit a regression of expenditures on assets and housing characteristics (equation 6), which we store as model M1. We then use M1 to predict the expenditures of all phone survey respondents based on asset ownership (roughly 1,000 respondents). We then draw a random sample (S2) with replacement from the phone survey respondents, and fit a regression of predicted expenditures on a variety of call metrics (equation 7), which we store as model M2. This model M2 is then used to predict the predicted expenditures of all of the mobile phone subscribers in our large dataset (roughly 1,000,000 individuals). We then draw a random sample (S3) with replacement from this population and estimate the wealth regression (equations 4 and 5). This process is repeated 1000 times. Each iteration yields a set of bootstrapped coefficients. We use the histogram of these bootstrapped coefficients to compute p-values. Specifically, the corrected p-values reported in the paper are, for each coefficient, the proportion of the histogram of the bootstrapped coefficients that is below 0 if the coefficient is positive, or above 0 if the coefficient is negative.
} 
top-ups is used as a proxy for individual wealth. In the phone survey data, we observe a strong positive correlation $(R=0.32)$ between this metric, which indicates the number of unique instances in a year when the individual deposited credit on his mobile phone account, and the wealth composite based on asset ownership. All coefficients have the expected sign. Similarly, Column 4 of Table 5 uses a measure of call activity that is negatively correlated with wealth, and we similarly observe that people who rank higher on this measure (i.e., who are poorer) receive fewer tranfers after the shock. The metric we construct is the "net incoming calls," which is a measure of the number of calls received by a subscriber minus the number of calls made by the subscriber, in the year 2007 (prior to the earthquake). This measure is negatively correlated with wealth $(R=-0.13)$, because of the cost structure of mobile phone calls in Rwanda. Whereas it costs money to make calls, it is free to receive calls. Thus, people who on balance receive more calls than they make tend to be poorer, whereas wealthy people who make more calls than they receive. Additional robustness tests for the wealth results are reported in Section 6.2(iv).

\section{(ii) Physical and Social Distance}

As discussed in the introduction, most favor exchange occurs in small, local communities. However, given the geographic pervasiveness of the mobile phone network, as well as the reduction in transaction costs associated with phone-based remittances, there is reason to suspect that the interpersonal transfers we study may not be similarly constrained by physical distance (Jack \& Suri 2014). Indeed, in our data we see empiricial evidence that a large portion of transfers are sent over long distances. Figure 4(a) shows the distribution of distances over which transfers are sent, for transactions involving individuals located in the earthquake region. While the vast majority of transfers are sent over a short distance, there are a large number of transfers sent to and from the capital of Kigali, which is approximately $150 \mathrm{~km}$ from the epicenter.

In response to the earthquake, there is further heterogeneity in transfers with respect to distance. The unconditional relationship between distance and transfers, both before and after the earthquake, is depicted in Figure 4(b). After the quake, the distribution shifts toward transfers occurring in an intermediate range of 20-130 kilometers. Presumably, this is because it is in that intermediate region where the other end of the dyad is likely to be unaffected by the quake, but still lives relatively close to $i$.

We estimate the magnitude of this effect of distance using a regression specification, which allows us to control for other dyad-specific factors that might be correlated with distance. For instance, the unconditional negative correlation between transfers and geographic distance is likely due partially to the fact that the the strength of social ties generally increases with physical proximity. Regression results, shown in Table 6 , indicate a negative association between distance and transfers, even when controlling for the "social proximity" between $i$ and $j$. In Table 6 , the social proximity of $i$ and $j$ is measured as the number of calls 
between $i$ and $j$; in Appendix Table 5, we use the number of distinct paths between $i$ and $j$ as a measure of tie strength (Karlan et al. 2009).

The negative relationship between physical distance and post-shock transfers is nonlinear. Figure 5 illustrates the nonparametric relationship between $\partial \tau_{i j} / \partial D_{i j}$ and $D_{i j}$ by plotting the coefficient estimates for $\beta_{3}$ from regression (5), where $Z_{i}$ is the number of contacts within $R$ kilometers, with a separate point for each $R$ from 0 to 250 . It is evident that, after the quake, people with many contacts near the epicenter do not receive more transfers. However, people with contacts more than $30 \mathrm{~km}$ away from the epicenter are more likely to receive transfers in the aftermath of the earthquake. Consistent with the earlier results of Figure 4(b), this effect dies down for contacts located more than $100 \mathrm{~km}$ from the epicenter.

\section{(iii) History Dependence}

Finally, we investigate the influence that the historical pattern of transfers between two individuals has on the transfers sent in response to the Lake Kivu earthquake. Specifically, we are interested in understanding whether there is evidence that individuals with a history of reciprocal transfers are more likely to exchange transfers.

As a starting point, we note that in equilibrium, the vast majority of pairwise relationships we observe do not exhibit a strong reciprocal component. Of the 646,713 dyadic pairs $(i, j)$ for which a transfer is observed in one direction (from either $i$ to $j$ or $j$ to $i$ ), transfers are observed in both directions in only 143,394 dyads (22 percent). We believe this normal pattern of transfers is indicative of the fact that, under normal circumstances, a great deal of phone-based giving is unidirectional, as from a worker remitting wages to family or a parent supporting a child. However, for the subset of dyadic pairs that are active in the 24-hour period following the earthquake, the ratio of unidirectional to bi-directional dyads jumps to 31 percent. The fact that bi-directional dyads are disproportionately represented in transfers associated with shocks suggests that past reciprocity may play a role in determining future transfers.

We test this formally in a regression using model (5), where past reciprocity is quantified by $T_{j i t}^{n e t}$, the net balance of payments made from $i$ to $j$ in the periods prior to $t$. Results are presented in Table 7 , with additional tests using the gross volume of transfers deferred to Appendix B. We observe that an individual $i$ who has a net positive balance with $j$ (i.e., $j$ "owes" $i$ money), is significantly more likely to receive help from $j$ on the day of the earthquake (row 2 of Table 7). Importantly, this effect persists even when controlling for the social proximity of $i$ and $j$, and is unlikely to be caused by unmodelled correlation between past transfer activity and general characteristics of the dyadic relationship (such as shared ethnicity, family ties, etc.), which are absorbed by the dyad fixed effects.

The positive and significant coefficient on $T_{j i t}^{n e t} * S h o c k_{i r t}$ is particularly striking given that the uninter- 
acted effect of the prior net balance is negative (row four Table 7). In other words, on normal days without shocks, transfers flow primarily in one direction: if $i$ has transferred more to $j$ than $j$ to $i$ prior to $t$, it is more likely that another $i$ to $j$ transfer will occur at $t$. This is consistent with the earlier observation that, under normal circumstances, there is a structural dependency where one person consistently gives and the other receives. However, in times of severe hardship, transfers flow in the opposite direction. After an economic shock, if $i$ has transferred more to $j$ than $j$ to $i$ prior to $t$, we are more likely to observe a transfer from $j$ to $i$.

\section{Discussion}

Do these effects matter? Based on the coefficient estimate in column (2) of Table 3, we observe that the earthquake produced an additional influx of roughly USD $\$ 6$ (2,800 RWF) to each of the 15 towers within $20 \mathrm{~km}$ of the epicenter, or approximately US\$84 (42,000 RWF) across all towers. In Panel C of Table 3, we further estimate that roughly US\$16,959 (8,479,935 RWF) was spent on phone calls to the affected area. Although this latter amount may represent an implicit transfer, since in Rwanda the caller bears the full cost of the call (all incoming calls are free), a more conservative interpretation focuses on the value of interpersonal airtime transfers.

The magnitude of these transfers, while large relative to normal behavior, is small in absolute terms. We emphasize the statistical significance of the effect, and the corresponding statistical significance of the heterogeneous effects described above, as we believe it is instructive in deepening our understanding of the complexion of mobile phone-based transfers following economic shocks. However, the literal economic significance of the transfers sent in response to this particular earthquake is likely small. The discrepancy between the statistical and economic significance is likely due in part to the low rates of uptake of the transfer service in early 2008. As can be seen in Figure 3, service utilization in Rwanda has increased significantly since the time of the earthquake. Prior to January 2008, when the quake occurred, only 1,400 individuals living in the earthquake region had ever used the airtime transfer service. The airtime transfer service subsequently gained more widespread adoption, creating a larger population of potential recipients, before being gradually superseded by mobile money, which was introduced in 2010. If airtime transfers had continued to increase proportionally to the number of active users, a similar earthquake in 2010 is predicted to have caused an influx of US\$25,000 to $\$ 33,000$ to affected areas. ${ }^{11}$ Of course, this extrapolation

\footnotetext{
${ }^{11}$ At the time of the earthquake, there were roughly 2,500 active mobile money users each day, in the whole of Rwanda. As of early 2010, according to communication with the operator, this number had grown to somewhere between 750,000 and $1,000,000$. Scaling transfers linearly with the increase in active users provides a lower bound of $\$ 25,200$. If traffic increases non-linearly in the number of subscribers, as much of the network literature suggests, the projected amount may even be much larger. Alternatively, if early adopters are not representative of late adopters and respond more strongly to an earthquake, these projections could represent upper bounds.
} 
is speculative and is not meant to distract from the small absolute value of transfers sent in response to the Lake Kivu earthquake.

There may be reason to suspect that in a time of severe shock, the marginal utility of an airtime transfer or an incoming phone call is higher than normal. As shown in Figure 6, most Rwandans carry very little airtime on their account - the median subscriber balance immediately prior to the earthquake was US $\$ 0.10$ (49.4 RWF), and roughly 32 percent of all subscribers had an airtime balance of less than UW $\$ 0.01$ (5 RWF), which is not sufficient to permit outgoing communication. In Rwanda, where an average phone call in 2008 lasted less than 30 seconds and cost roughly US $\$ 0.08$ (40 RWF), a small transfer could thus be sufficient to enable the recipient to make a phone call or send a text message. Such a transfer would also enable a recipient with zero balance to initiate a "missed call," where a caller dials a number but hangs up before the recipient answers. In Rwanda and many other locations this is a common way of communicating when the caller wishes to talk but does not want to pay for the cost of a call. Sending missed calls in Rwanda in 2008 required that the subscriber have a positive balance on his or her account.

In other contexts, such communications have been instrumental in facilitating relief efforts. ${ }^{12}$ In Rwanda, we do not know how these transfers were used, whether to call for help, to coordinate relief efforts, or simply to reassure a loved one. We do, however, observe that the recipients of transfers were disproportionately likely to use the credit immediately. For instance, roughly $70 \%$ of recipients made a call within 24 hours of receiving the quake-induced transfers. On normal days, the corresponding rate is $22 \%$.

On the other hand, since what we observe is transfers of airtime, not cash, and to the extent that the marginal utility of airtime is lower than the marginal utility of cash, the realized benefit of these transfers could be even smaller than the point estimates suggest. For instance, if the sole benefit of this transfer were infra-marginal savings on future airtime expenditures, our estimates should at least be deflated by the informal $20 \%$ commission charged for converting airtime to cash, plus additional transaction costs. Since we only observe activity that occurs on the mobile phone networks, it is impossible for us to ascertain whether these transfers allowed the recipients to smooth consumption, as traditional models of risk sharing predict. We are similarly unable to infer whether the mobile phone-based transfers are substitutes for transfers that would have otherwise been sent using another mechanism, or whether they affect the extensive margin. If the advantages of the technology (speed, efficiency, lowered transaction costs, and lowered minimum transaction) induce more people to give, we might observe a net increase in total transfers. Alternatively, it is possible that mobile phone-based transfers, which tend to be quite small (usually on the order of one dollar), could

\footnotetext{
${ }^{12}$ For a recent example, see "In Turkey, Desperate Race to Find Trapped Survivors", New York Times, October 25, 2011: "Some dug with their bare hands, while other used heavy machinery to remove chunks of fallen concrete and relied on cellphone calls from the missing in the search for survivors... a 19-year-old in the town survived by using his cellphone to direct teams to the collapsed building where he had been trapped."
} 
crowd out other gifts that would otherwise have been sent in a larger denomination.

\subsection{Motives for Mobile Phone-Based Giving}

We have thus far shown that severe economic shocks produce a small but significant increase in transfers sent over the Rwandan mobile phone network to individuals affected by catastrophic shocks. We interpret this effect as prima facie evidence that people use the mobile network to help each other cope with economic shocks. We have also observed three forms of heterogeneity in the period immediately following an economic shock: that wealthy individuals receive more; that transfers decrease with geographic distance; and that past reciprocity is predictive of future transfers.

Before concluding, we briefly investigate the possible motives that might underlie these transfers. In particular, we wish to determine whether the set of empirical findings are consistent with a simple model of pro-social behavior, or whether they are best interpreted as three separate forms of heterogeneity. To this end, we contrast two stylized models of prosocial behavior, which, following Leider et al. (2009), we call 'charity' and 'reciprocity. ${ }^{\text {'13 }}$ This discussion is admittedly speculative, as our investigation is constrained by the available data. ${ }^{14}$

\section{(i) Stylized models of charity and conditional reciprocity}

By charity we refer to the broad class of motives where a giver gives because he receives direct utility from the act of giving or from increasing the utility of another. The canonical example of this behavior is pure altruism, where one person's utility depends positively on another's (Becker 1976, Andreoni \& Miller 2002):

$$
U_{i t}=u_{i}\left(x_{i t}-\tau_{j i t}\right)+\gamma_{i j} u_{j}\left(x_{j}+\tau_{j i t}\right)
$$

As before, we denote by $\tau_{j i t}$ a transfer sent from $i$ to $j$ at time $t$. The larger the parameter $\gamma_{i j}$, the more $i$ values $j$ 's utility. Alternatively, $\gamma_{i j}$ can be seen as representing an unconditional sharing norm that dictates a transfer from $i$ to $j$.

We contrast this idea to that of conditional reciprocity. By this, we refer to motives that are embedded in conditional sharing norms and long-term relationships. Here, the exchange of favors is motivated by - or conditioned on - the expectation of future reciprocation (cf. Coate \& Ravallion 1993, Karlan et al. 2009). This modeling framework offers the advantage that it most transparently leads to empirical predictions that

\footnotetext{
${ }^{13}$ Our distinction also parallels the distinction Ligon \& Schechter (2011) draw between "preference-related" motives and "incentive-related" motives.

${ }^{14}$ For instance, we stop well short of recent experimental work that, through clever manipulation of experimental conditions, can differentiate between different types of reciprocity (Ligon \& Schechter 2011, Cabral et al. 2011, Leider et al. 2009, Charness \& Rabin 2002). See also Kinnan (2014) for an empirical test between barriers to risk sharing in rural villages.
} 
can be tested with the data at our disposal. One way of capturing this idea comes out of economic theory, and is best examplified by the dynamic limited commitment model developed by Kocherlakota (1996) and Ligon et al. (2002). Models of conditional reciprocity have also been proposed by experimentalists. One such model is the intrinsic, preference-based model of Rabin (1993) and Falk \& Fischbacher (2006). ${ }^{15}$ The two modeling framework differ in some important details, but they make predictions that are observationally equivalent for the data at our disposal, so we ignore these differences here.

The limited commitment model of Kocherlakota (1996) and Foster \& Rosenzweig (2001) provides a convenient illustration of the main idea of conditional reciprocity. Imagine that individual $i$ has stationary, single-period utility specified by (8). Now add the idea that $i$ expects to benefit from future interaction with $j:$

$$
U_{i t}=u_{i}\left(x_{i t}-\tau_{j i t}\right)+\gamma_{i j} u_{j}\left(x_{j t}+\tau_{j i t}\right)+E \sum_{s=t+1}^{\infty} \delta^{s-t}\left[u_{i}\left(x_{i s}-\tau_{j i s}\right)+\gamma_{i j} u_{j}\left(x_{j s}+\tau_{j i s}\right)\right]
$$

The first part of (9) is identical to the altruistic model (8). The second term captures the discounted expected utility of the relationship, which is the expected value of future reciprocation. When $\gamma_{i j}$ is small or zero, the exchange of favors is constrained by what $i$ expects to receive from $j$ in the future. In a cooperative equilibrium, $i$ and $j$ will be observed exchanging favors over time; if one of them stops, the other will stop as well to retaliate. The behavior of $i$ and $j$ that is predicted by this model is thus observationally similar to the conditional reciprocity concept of Rabin (1993) and Falk \& Fischbacher (2006).

\section{(ii) Empirical predictions and results}

For the types of heterogeneity which we have tested with our data, these two models yield different empirical predictions. These predictions, as well as the sign of the corresponding coefficients estimated in our data, are given in Table 8. The comparative statics are derived in Appendix A, but the intuition is straightforward.

1. Wealth: If transfers are motivated by charity, they are expected to flow from wealthier to poorer individuals as the marginal utility of the transfer is likely to be higher for a poorer individual. This may be particularly true in Rwanda, where poorer individuals are significantly more likely to carry a zero-balance on their account, which prevents them from making an outgoing call or sending a text message. ${ }^{16}$ A model of reciprocity, by contrast, is more ambiguous with respect to the weath of the recipient. If anything, we might expect wealthier individuals to receive more, as the continuation value of a relationship is, all else equal, higher with a wealthy person whose participation constraint is less

\footnotetext{
${ }^{15}$ This literature has sought to differentiate between the different types of reciprocity. For recent experimental work that differentiates between different types of reciprocity, see Leider et al. (2009), Ligon \& Schechter (2011), and Cabral et al. (2011). Fehr \& Schmidt (2006) and Sobel (2005) provide theoretical overviews. We cannot test these different concepts of reciprocity with the observational data that we have.

${ }^{16}$ The correlation between end-of-day balance and wealth is 0.15 , with a T-statistic of 58.8 . See Figure 6 .
} 
likely to bind in the future. Our results indicate that earthquake-induced transfers increase in the wealth of the recipient $\left(x_{i}\right)$ but are not significantly correlated with the wealth of the sender $\left(x_{j}\right)$. This finding is consistent with a conditional reciprocity model of favor exchange, but harder to explain as motivated by charity. On its own, however, this evidence is not particularly compelling.

2. Geographic distance: Several factors could in principle lead to the observed negative correlation between distance and transfers. But the particular non-linear form of this relationship can easily be accounted by a model of favor exchange under conditional reciprocity (see Appendix A.2). In particular, when $i$ and $j$ live close to one another, they are likely to be affected in the same way by large, covariate shocks, and thus less able to help each other than they would in response to smaller, idiosyncratic shocks. This is precisely what we see in the data, with fewer transfers flowing within the earthquake region, and most of the increase coming from individuals $20 \mathrm{~km}$ or more from the recipient (Figure 4). Outside of the affected region, we then observe a slow decrease as distance increases, which would be expected if monitoring and enforcement costs increase with physical distance. In contrast, a simple model of charity predicts no direct effect of physical distance on transfers. To explain our findings within the framework of a charity model, altruism would have to vary systematically with distance in the way observed in the data. One possibility, which we cannot rule out, is that altruism is influenced by ethnic or regional identity, and the sense of shared identity falls systematically with distance.We discuss some of these alternative explanations in Section 6.1 (iii) below.

3. History-dependence: Finally, as derived in Appendix A.3, conditional reciprocity makes a specific prediction with respect to past transfers that is consistent with the data: $j$ is expected to make a smaller transfer to $i$ following a shock to $i$ when the net balance of $i$ - $j$ transfers is negative, i.e., when $i$ has received more from $j$ than $j$ has received from $i$ in the recent past. This formal property is intuitive: a negative balance of transfers, which we measure as $T_{i j t}^{n e t}$, can be thought of as the amount that $i$ "owes" $j$ at the time of the shock. By contrast, if shock-induced transfers are motivated by pure charity, there is no obvious reason why they should depend on prior transfers. In fact, to the extent that past transfers from $j$ to $i$ signal $j$ 's altruism towards $i$, transfers sent in response to the earthquake should, if anything, be increasing in past transfers from $j$ to $i$. We find the opposite. ${ }^{17}$ Furthermore, conditional reciprocity also implies that a positive $T_{i j t}^{n e t}$ should not affect transfers following a shock to $i$. The intuitive logic is the same: if $i$ does not "owe" anything to $j$, what $j$ is willing to give to $i$ is only bound by $j$ 's voluntary participation constraint which, in this case, is unlikely to be binding. We test this specific prediction in Appendix Table 9 by estimating the regression separately

\footnotetext{
${ }^{17}$ One possibility we test for is that those who have transferred a lot of airtime to others are likely to have a low available balance and therefore a high marginal utility of airtime. In results not shown, we observe that controlling for the end-of-day balance before the earthquake does not impact our estimates of the effect of $T_{i j t}^{n e t}$ on transfers.
} 
for positive and negative $T_{i j t}^{n e t}$. As predicted by the reciprocity model, the coefficient of $T_{i j t}^{n e t} * S h o c k_{i r t}$ is significant only when $T_{j i t}^{\text {net }}$ is negative, not when it is positive.

Each of the above findings is consistent with the model of conditional reciprocity (9). This is not to say that each of these findings cannot be reconciled with the charity model (8) by introducing alternative explanations. Indeed, we discuss some of these alternative explanations below. Yet, as no single explanation is capable of explaining all our findings, Occam's razor suggests leaning towards the model that most simply explains all the findings taken together, even if the evidence cannot be regarded as definitive.

\section{(iii) Alternative explanations}

Above, we have argued that the full set of empirical results appears more consistent with a simple model of conditional reciprocity than a simple model of charity. This interpretation relies on the heterogeneity of the transfers with respect to the wealth of the recipient, the physical distance of the dyad, and the past history of transfers. For each of these findings, alternative explanations exist. We discuss those briefly here. In Section 6.2 below, we will show that the findings themselves are robust.

A few of the possible alternative explanations for the observed heterogeneity with respect to wealth have already been discussed. For instance, under a model of charity, wealthier individuals might be expected to receive more if they were disproportionately affected by the quake. However, as discussed in Section 5, the limited qualitative evidence that we can find suggests that people at different wealth levels were equally likely to be affected (USGS 2009). While wealthy individuals are more likely to own assets that could be affected by the earthquake, we can find nothing that would indicate that the relative damage suffered by wealthy people was any greater than that of poor people. Besides, airtime transfers were well below the value of the damage suffered, so they could not be construed as compensating for damages, more as a way to help in an emergency.

A related concern is that the marginal utility of airtime consumption may be higher for the wealthy because they consume more phone services. Normally we would expect charitable donations to be directed towards the less fortunate members of society. But when charitable assistance is provided in kind, one could argue that it should take the form most valued by recipients. Hence, since the wealthy consume more phone services, they should be more likely to receive in-kind assistance in the form of phone airtime. While we cannot fully rule out this possibility, we find it quite unconvincing in our case. The response to the earthquake that we study is the immediate response, within a short time interval after the earthquake. Within this time interval, sending airtime was pretty much the only form of material assistance that households elsewhere in the country could provide on a peer-to-peer basis (see Table 1). If it is the only form of individual 
charity possible during this period, we would not expect most of it to go to the wealthiest phone owners. Furthermore, as discussed in Section 6, on the day of the earthquake the marginal utility of airtime was, if anything, probably higher for poor people because they are more likely to carry a zero-balance on their phone account.

An additional factor, independent of conditional reciprocity, which may account for the wealth result would be if wealthy individuals receive more transfers because they function as intermediaries who are expected to redistribute such transfers to other people in the area, rather than utilize the airtime themselves. We cannot entirely rule out this explanation, if subsequent transfers occur off of the mobile phone network. However, such redistribution does not occur on the mobile phone network. As noted above, it appears that the primary use of the transfer is to make an immediate phone call, with roughly $70 \%$ of the post-earthquake recipients placing a call within 24 hours of receiving a transfer.

The fact that transfers decrease with physical distance may be caused by unrelated factors, such as the fact that people care more about people who live closer by, for instance because those people have similar characteristics. In our econometric specifications, we address this concern in two ways: first, by including dyad-specific fixed effects, we control for unobserved heterogeneity for each pair of individuals. In practice, this means that any fixed characteristic of the dyad (for instance, the possibility that $i$ has a strong affinity for $j$ and consistently sends $j$ transfers) is absorbed in the fixed effect. The primary coefficient of interest reflects the additional transfer sent between geographically-proximate individuals in response to shocks, after accounting for any heightened transfers at baseline. Second, by controlling for a variety of measures of social

proximity $S_{i j}$, we attempt to isolate the effect of geographic distance on shock-induced transfers, conditional on social distance. Factors such as kinship, co-ethnicity, and other forms of homophily are likely to be captured in this regressor.

The history-dependence that we observe is perhaps the strongest indication that reciprocity is at play. To restate the result: we find that in normal circumstances, transfers flow primarily in a single direction, and $i$ is more likely to receive from $j$ if $i$ has received from $j$ in the past. However, after an economic shock, the opposite trend exists: $i$ is less likely to receive from $j$ if $i$ has received more from $j$ in the past. It is difficult to come up with a reasoning that would account for this finding within the framework of a charity model.

\subsection{Robustness}

While the vast quantity of observations in our regressions makes it possible to detect relatively small changes in transfer activity, we conduct a large number of robustness checks to ensure that the results are not a mere 
artifact of our dataset. Here, we present the results from a series of "placebo" tests to demonstrate that similar results do not obtain on days where no earthquake occurs (Table 9), and that the inclusion of lag and lead terms does not alter the results (Table 10). To demonstrate that the effects observed in response to the Lake Kivu earthquake are likely to generalize to other severe shocks, we show that a similar, albeit muted, response is observed after a series of large floods that occurred in late 2007 (Table 11). Finally, in section 6.2 we discuss possible endogeneity and limitations of the proxy we construct to measure SES.

In Appendix B, we further show that the our results are not sensitive to the econometric specification, including the use of fixed effects or the inclusion of different time-varying controls (Appendix B.1). To address the possibility that our effects are driven in part by outliers or a dependent variable with a long right tail, Appendix B.2 uses binarized, winsorized, and other simple transformations of the dependent variable that are less sensitive to outliers. We also provide evidence that our results are not affected by the structure imposed on the variance matrix, including a variety of alternative methods for clustering standard errors (Appendix B.3); that they do not depend on the choice of time window being analyzed (also Appendix B.1) or on any specific measure of social proximity (Appendix B.4); and that it is unlikely that our heterogeneous effects are driven by mean reversion (Appendix Table 9) .

\section{(i) Placebo tests}

As a robustness check of the average treatment effect, we verify that the effects of the earthquake on transfers are unique to the day of the earthquake, and do not generally occur on days without significant economic shocks. We do this first at the district level, following the methodology used to produce Table 3. In Appendix Table 10, we include lag and lead terms to test whether there was a significant effect of the earthquake on transfer patterns in the days immediately before and after the earthquake. To identify these ten additional terms, we include district-level data from the full dataset as in Appendix Table 1. In column 1, we observe that this effect does not exist, and before the earthquake (lead1-lead3) and after the earthquake (lag1-lag7), there was no significant change in transfers to the affected regions. These results hold for lags and leads of up to 10 days. In columns (2) and (3), we see in contrast that national calls to the affected region increase in the days following the earthquake. International calls do not. Critically, there was no anomalous increase in any sort of mobile network traffic in the days prior to the earthquake.

Table 9 presents results from testing the same specification as in column 4 of Table 3 but with a "placebo" shock at the same location on different dates. Thus, we test for a spurious effect several months before (columns 1-3) and after (column 5) the actual earthquake (column 4). In contrast to the results obtained for the date of the actual earthquake, we observe no significant change in transfers on the day of the placebo earthquakes. 


\section{(ii) Other large covariate shocks}

The results presented so far provide strong evidence that Rwandans used the mobile phone network to send airtime to friends and families affected by a major earthquake, and that these results are robust to different empirical specifications. We now show that similar transfers are observed following other natural disasters.

During the period for which we have mobile phone data, there were no massive natural disasters on the scale of the Lake Kivu earthquake. However, there were two major floods that severely disrupted the lives of many Rwandans. These floods are not as well suited to our estimation strategy as the earthquake, since floods are less precisely located in space (there is no single epicenter), and the timing is only partially exogenous (prior weather patterns anticipate floods). Therefore, there are a priori reasons to expect that the effect of a flood on transfers would be less pronounced than the effect of an earthquake.

Nonetheless, we do observe a significant increase in transfers on the days following a severe flood. In Appendix Table 11, we estimate equation (1) for the towers in the region of a flood that killed 17 during September 2007. We find a modest but strongly significant increase in airtime sent to regions affected by the flood. In column (4) of Table 11, the point estimate is roughly half that of the corresponding point estimate of the effect of the earthquake (column 4 of Table 1).

\section{(iii) Net vs. gross transfers}

As a further robustness check, we repeat the same analysis using net instead of gross transfers. The concern is that gross transfers may misrepresent the aggregate magnitude of the transfers if individuals who receive airtime pass it on to others in the same region. This could result in double-counting at the district or cell tower level. Thus, we redefine the dependent variable in regression (2) as $\tau_{i r t}^{\prime}=\sum_{j} \tau_{i j r t}-\sum_{j} \tau_{j i r t}$, that is, the transfers received by $i$ from others minus the transfers given by $i$ to others. At the district and cell tower

levels, we similarly redefine Let $\tau_{r_{1} r_{2} t}=\sum_{i \in r_{1}} \sum_{j \in r_{2}} \tau_{i j r t}$ where $r_{1}$ and $r_{2}$ are two different locations (e.g., districts or cell tower area); $\tau_{r_{1} r_{2} t}$ represents the total transfers received by individuals in location $r_{1}$ from individuals in location $r_{2}$. Summing over all other locations yields the gross transfers from other locations to location $r_{1}$. Net inflows to region $r_{1}$ are thus $\tau_{r_{1} t}^{\prime}=\sum_{r_{2}} \tau_{r_{1} r_{2} t}-\sum_{r_{2}} \tau_{r_{2} r_{1} t}$. Results, shown in Panel B of Table 3 are similar in significance and magnitude to those reported in Panel A, implying that the magnitude of our findings is not driven by double counting.

\section{(iv) Limitations with our measure of inferred wealth}

Estimation of models (4) and (5) relies on a proxy for SES, $x_{i}$, to measure the relative socioeconomic status of the population of mobile phone subscribers. In utilizing the $\widehat{\widehat{Y_{i d}}}$ as computed above, one potential concern 
is that our $x_{i}$ is more a proxy of aggregate phone use or technological sophistication than actual economic wealth. We take several steps to minimize this possibility.

Two precautions have been discussed already: First, as noted in Section 5.2 (i), we find qualitatively similar results when using a variety of more parsimonious proxies for wealth. In particular, whether using a simple metric that is likely to be postively correlated with wealth (i.e., total recharges) or negatively correlated with wealth (i.e., net incoming calls), we find that the heterogeneous effect persists with the expected sign. Second, when estimating (7) we exclude all metrics related to use of the airtime transfer service. This helps reduce the primary source of endogeneity, that there exists a mechanical correlation between $\widehat{\widehat{Y_{i d}}}$ and $\tau_{i j r t}$.

Third, we use a highly non-parametric model to estimate (7) that includes district fixed effects and second-order polynomial terms. As a result, there are strong non-monotonicities in (7) such that $\widehat{Y_{i d}}$ is increasing in some measures of phone use, decreasing in others, and in general is highly non-linear. This ameliorates the concern that $\widehat{\widehat{Y_{i d}}}$ simply captures aggregate phone use.

Fourth, we explicitly control for different measures of aggregate phone use in our regressions that include $\widehat{\widehat{Y_{i d}}}$. Column 2 of Table 5 presents the results of re-estimating the partial effect of wealth on transfers received (Table 4 in the main text, reproduced as column 1 of 5), while additionally controlling for "Recipient Calling Activity," which is simply the total number of calls made by the recipient in 2007. We assume that this measure is likely to be more directly correlated with the recipient's general propensity to utilize the phone than our non-parametric measure of wealth $x_{i}=\widehat{\widehat{Y_{i d}}}$. Comparing the results of columns 1 and 2 of Table 5 , we note that all coefficients have the same sign and statistical significance. In particular, the correlation between recipient wealth and transfers received remains positive and significant. Separately controlling for additional measures of calling activity, such as the sum of calls made and calls recieved, produces very similar results.

Beyond these concerns which we can address explicitly, there are additional limitations to the approach we have taken to estimating wealth from call records over which we have less control. For instance, we have glossed over distinctions between income, expenditures, permanent income, and wealth, which sometimes are of material consequence (cf. Deaton \& Muellbauer 1980). Also problematic is the possibility that asset-based proxies for expenditures may provide biased estimates of the expenditures of certain types of individuals. For instance, if a strong correlation is found between television ownership and assets among the aggregate population, but a small subgroup of the population has a distaste for television, this simple method would systematically underestimate the expenditures of that subgroup. Finally, one particularly troubling assumption we must make is that the relationship between assets and expenditures identified with the function $f(\cdot)$ in the 2005 RHS data will remain constant when applied to phone survey data collected in 
$2009 .{ }^{18}$ By using $\widehat{\widehat{Y_{i d}}}$ as a proxy for $x_{i}$, we do not mean to imply that this method provides an externally valid measure of an individual's wealth, or that $\widehat{\widehat{Y_{i d}}}$ could be used to infer changes over time. Rather, we intend it to provide a proxy for the relative wealth of individuals in our mobile phone dataset so as to be able to assess heterogeneity among our population of interest.

\section{Conclusion}

Analyzing four years of activity on Rwanda's largest mobile phone network, we have tested whether the mobile phone network is used to transfer resources to individuals affected by natural disasters. We find a significant increase in airtime transferred to individuals affected by covariate shocks, and a large increase in expenditures on communication to those same individuals. Given the low rates of adoption of the airtime transfer service at the time of the shocks, the absolute value of money transferred is small, but simple a backof-the-envelope calculation indicates that just a few years later, the total response to a similar earthquake in Rwanda might have been as large as $\$ 25,000$ to $\$ 33,000$.

The average response masks significant heterogeneity: wealthier individuals and individuals with contacts near the earthquake epicenter are more likely to receive transfers. This heterogeneity sheds some light on the motives behind these transfers. We seek to reconcile our results with two canonical models of giving, one based on pure charity, the other on conditional reciprocity. We show that these two motives for giving produce conflicting empirical predictions, in particular with respect to the marginal effect of past transfers, wealth, and geographic distance. Testing these hypotheses with the data from Rwanda, we find that the pattern of findings is consistent with the predictions of the conditional reciprocity model, but is harder to reconcile with a pure charity model.

Given the increasing prominence of mobile technology in developing countries, it is important to develop a better understanding of the economic impacts that this technology will have on the lives of its users. In this paper, we argue that mobile phones can provide a new method for coping with unexpected shocks by facilitating communication and enabling long-distance interpersonal transfers. Since the alternative mechanisms used for remittances are considerably slower and more expensive, this immediate influx of support may be of material consequence.

In the time that has passed since the 2008 earthquake, the landscape of mobile phone use in Rwanda

\footnotetext{
${ }^{18}$ This assumption is unjustified for at least two distinct reasons. First, the data for the two populations was collected using very different methodologies, and respondents may respond differently to questions about assets depending on whether they are asked in person or over the phone. Second, the data was collected in different years, and it is possible that the relationship between assets and expenditures would evolve over such a long interval. For instance, the strong relationship observed in 2005 between television ownership and wealth may be weaker in 2009, as electricity becomes more available and used televisions saturate the market.
} 
has changed in important ways. The number of mobile-cellular telephone subscriptions per 100 inhabitants has climbed from less than 1 in 2002, to roughly 15 at the time of the earthquake, to nearly 57 in 2013 (ITU 2014). ${ }^{19}$ Thus, while the average mobile phone user in 2008 was roughly half as wealthy as the average Rwandan citizen in 2008 (Blumenstock \& Eagle 2012), today the mobile phone subscriber base is more representative of the adult population of the country. Given the low transactions costs of phone-based transfers relative to existing alternatives, we expect the potential benefits of the technology to continue to increase.

Over the past several years, the primary Rwandan operator and several of its competitors have upgraded their network from a basic system for transferring airtime to a set of fully- fledged mobile money networks. Similar in features to the wildly popular M-Pesa network in neighboring Kenya, Rwandan mobile money networks allow for over-the-counter purchases, official re-conversion of airtime to cash by authorized agents, and several other value-added services. There are currently over one million active users of the mobile money network in Rwanda (Argent et al. 2013). While we do not have data on mobile money usage, part of the reason why uptake has been so rapid in East Africa is that it functions as a system for remitting money to people located elsewhere. This type of usage is analogous to the airtime transfer system we study. Thus, if the use of mobile money continues to spread, its scope for facilitating risk sharing across space will similarly continue to grow. Our study nevertheless suggests that the potential benefits of mobile technology may not be evenly distributed. There is a sharp divide between people who do and don't own mobile phones, and as we show, it is the wealthiest who are most likely to reap the benefits of phone-based transfers. Such evidence suggests that policies that more actively target poorer segments of the population, and which lower barriers to adoption and use, might better ensure that the potential benefits of mobile phones are realized by those with the greatest need.

\footnotetext{
${ }^{19}$ Note that this particular metric of mobile phone penetration is an overestimate of the number of unique individuals, as many individuals have multiple SIM cards registered to their name.
} 


\section{References}

Aker, J. (2008), 'Does digital divide or provide? the impact of cell phones on grain markets in Niger', $B R E A D$ Working Paper (177).

Aker, J. C. \& Fafchamps, M. (2014), 'Mobile Phone Coverage and Producer Markets: Evidence from West Africa', The World Bank Economic Review p. lhu006.

*http://wber.oxfordjournals.org/content/early/2014/10/08/wber.lhu006

Aker, J. C. \& Mbiti, I. M. (2010), 'Mobile Phones and Economic Development in Africa', Journal of Economic Perspectives 24(3), 207-232.

*http://www.aeaweb.org/articles.php?doi=10.1257/jep.24.3.207

Andreoni, J. (1990), 'Impure altruism and donations to public goods: a theory of warm-glow giving', The Economic Journal 100(401), 464477.

Andreoni, J. (2006), 'Philanthropy', Handbook on the Economics of Giving, Reciprocity and Altruism 2, 12011269.

Andreoni, J. \& Miller, J. (2002), 'Giving according to GARP: an experimental test of the consistency of preferences for altruism', Econometrica 70(2), 737-753. ArticleType: research-article / Full publication date: Mar., 2002 / Copyright 2002 The Econometric Society.

Argent, J., Hanson, J. \& Gomez, M. P. (2013), 'The regulation of mobile money in rwanda', International Growth Centre Working paper.

Becker, G. S. (1974), 'A theory of social interactions', Journal of Political Economy 82(6), 1063-1093.

Becker, G. S. (1976), 'Altruism, egoism, and genetic fitness: Economics and sociobiology', Journal of Economic Literature 14(3), 817826.

Bertrand, M., Duflo, E. \& Mullainathan, S. (2004), 'How much should we trust Differences-in-Differences estimates?', Quarterly Journal of Economics 119(1), 249275.

Blumenstock, J., Callen, M. \& Ghani, T. (2015), 'Mobile-izing Savings: Experimental Evidence on the Impact of Automatic Contributions in Afghanistan'.

Blumenstock, J. E. \& Eagle, N. (2010), 'Mobile divides: Gender, socioeconomic status, and mobile phone use in Rwanda', 4th International IEEE/ACM Conference on Information and Communications Technologies and Development.

Blumenstock, J. E. \& Eagle, N. (2012), 'Divided we call: Disparities in access and use of mobile phones in Rwanda', Information Technology and International Development 8(2), 1-16.

Bolton, G. E. \& Ockenfels, A. (2000), 'ERC: a theory of equity, reciprocity, and competition', American Economic Review p. 166193. 
Burbidge, J. B., Magee, L. \& Robb, A. L. (1988), 'Alternative transformations to handle extreme values of the dependent variable', Journal of the American Statistical Association 83(401), 123127.

*http://www.tandfonline.com/doi/abs/10.1080/01621459.1988.10478575

Cabral, L., Ozbay, E. \& Schotter, A. (2011), Intrinsic and instrumental reciprocity: An experimental study, Technical report, Working paper). New York University.

CGAP and GSMA (2009), 'Mobile money for the unbanked: Annual report 2009'.

Charness, G. \& Rabin, M. (2002), 'Understanding social preferences with simple tests*', Quarterly journal of Economics 117(3), 817869 .

Coate, S. \& Ravallion, M. (1993), 'Reciprocity without commitment : Characterization and performance of informal insurance arrangements', Journal of Development Economics 40(1), 1-24.

Collins, D., Morduch, J., Rutherford, S. \& Ruthven, O. (2009), Portfolios of the Poor: How the World's Poor Live on \$2 a Day, Princeton University Press.

De Vreyer, P., Gubert, F. \& Roubaud, F. (2010), 'Migration, self-selection and returns to education in the WAEMU', Journal of African Economies 19(1), $52-87$.

de Weerdt, J. \& Fafchamps, M. (2010), 'Social identity and the formation of health insurance networks', Working Paper.

Deaton, A. \& Muellbauer, J. (1980), Economics and consumer behavior, Cambridge Univ Pr.

Deaton, A. \& Zaidi, S. (2002), Guidelines for constructing consumption aggregates for welfare analysis, World Bank Publications.

Donner, J. (2008), 'The rules of beeping: Exchanging messages via intentional "Missed calls" on mobile phones', Journal of Computer-Mediated Communication 13(1), 1-22.

Fafchamps, M. (1999), 'Risk sharing and quasi-credit', Journal of International Trade and Economic Development 8, 257278 .

Fafchamps, M. \& Gubert, F. (2007), 'The formation of risk sharing networks', Journal of Development Economics 83(2), 326-350.

Fafchamps, M. \& Lund, S. (2003), 'Risk-sharing networks in rural philippines', Journal of Development Economics $\mathbf{7 1}(2), 261-287$.

Fafchamps, M. \& Minten, B. (2012), 'Impact of SMS-Based Agricultural Information on Indian Farmers', The World Bank Economic Review 26(3), 383-414.

Falk, A. \& Fischbacher, U. (2006), 'A theory of reciprocity', Games and Economic Behavior 54(2), 293-315.

Fehr, E. \& Schmidt, K. M. (1999), 'A theory of fairness, competition, and cooperation*', Quarterly journal of Economics 114(3), 817868. 
Fehr, E. \& Schmidt, K. M. (2006), 'The economics of fairness, reciprocity and altruism-Experimental evidence and new theories', Handbook on the economics of giving, reciprocity and altruism 1, 615691.

Foster, A. D. \& Rosenzweig, M. R. (2001), 'Imperfect commitment, altruism, and the family: Evidence from transfer behavior in Low-Income rural areas', Review of Economics and Statistics 83(3), 389-407.

Fox, J. (1997), Applied regression analysis, linear models, and related methods, Sage Publications, Inc.

Genicot, G. \& Ray, D. (2003), 'Group formation in RiskSharing arrangements', Review of Economic Studies 70(1), 87-113.

Gine, X. \& Yang, D. (2009), 'Insurance, credit, and technology adoption: Field experimental evidencefrom malawi', Journal of Development Economics 89(1), 1-11.

GSMA (2014), 'MMU deployment tracker | mobile for development'.

*http://www.gsma.com/mobilefordevelopment/programmes/mobile-money-for-the-unbanked/insights/tracker

Hoffman, M. (2010), 'Does higher income make you more altruistic? evidence from the holocaust', Review of Economics and Statistics 93(3), 876-887.

Jack, W. \& Suri, T. (2012), 'Risk sharing and transaction costs: Evidence from Kenya's mobile money revolution', Working Paper.

Jack, W. \& Suri, T. (2014), 'Risk sharing and transactions costs: Evidence from kenya's mobile money revolution', American Economic Review 104(1), 183-223.

*http://www.aeaweb.org/articles.php?doi=10.1257/aer.104.1.183

Jensen, R. (2007), 'The digital provide: Information (Technology), market performance, and welfare in the south indian fisheries sector', The Quarterly Journal of Economics 122(3), 879-924.

Kabbucho, K., Sander, C. \& Mukwana, P. (2003), 'PASSING THE BUCK: money transfer systems: The practice and potential for products in Kenya', MicroSave White Paper.

Karlan, D., Mobius, M., Rosenblat, T. \& Szeidl, A. (2009), 'Trust and social collateral', The Quarterly Journal of Economics 124(3), $1307-1361$.

Kinnan, C. (2014), 'Distinguishing barriers to insurance in thai villages', Working Paper .

Leider, S., Mbius, M. M., Rosenblat, T. \& Do, Q. (2009), 'Directed altruism and enforced reciprocity in social networks', The Quarterly Journal of Economics 124(4), 1815 -1851.

Ligon, E. (1998), 'Risk sharing and information in village economies', The Review of Economic Studies 65(4), 847864.

Ligon, E. A. \& Schechter, L. (2011), 'Motives for sharing in social networks', SSRN eLibrary .

Ligon, E., Thomas, J. P. \& Worrall, T. (2002), 'Informal insurance arrangements with limited commitment: Theory and evidence from village economies', The Review of Economic Studies 69(1), 209-244. 
List, J. A. \& Lucking-Reiley, D. (2002), 'The effects of seed money and refunds on charitable giving: Experimental evidence from a university capital campaign', Journal of Political Economy 110(1), 215233.

Marmaros, D. \& Sacerdote, B. (2006), 'How do friendships form?', Quarterly Journal of Economics 121(1), 79119.

Mas, I. \& Mayer, C. (2011), 'Savings as Forward Payments: Innovations on Mobile Money Platforms', Available at SSRN 1825122.

Mbiti, I. \& Weil, D. N. (2011), Mobile banking: The impact of M-Pesa in Kenya, Technical report, National Bureau of Economic Research.

*http://www.nber.org/papers/w17129

McKay, C. \& Pickens, M. (2010), 'Branchless banking 2010: Whos Served? At What Price? Whats Next?', Washington, D.C. .

Montgomery, M. R., Gragnolati, M., Burke, K. A. \& Paredes, E. (2000), 'Measuring living standards with proxy variables', Demography 37(2), 155174.

Murphy, K. M. \& Topel, R. H. (2002), 'Estimation and inference in two-step econometric models', Journal of Business 85 Economic Statistics 20(1), 88-97.

*http://amstat.tandfonline.com/doi/full/10.1198/073500102753410417

National Institute of Statistics-Rwanda (2007), Enqute Intgrale sur les Conditions de Vie des Mnages 2005-2006 (EICV2005, v.1.1), INSR and ORC Macro, Calverton, Maryland.

Orozco, M. (2009), 'Emerging markets for Rwanda: remittance transfers, its marketplace and financial intermediation'.

Platteau, J. P. (1995), 'An indian model of aristocratic patronage', Oxford Economic Papers p. 636662.

Pulver, C. (2009), 'The performance and impact of MPESA: preliminary evidence from a household survey', Presentation to FSD-Kenya .

Rabin, M. (1993), 'Incorporating fairness into game theory and economics', The American Economic Review p. 12811302 .

Republic of Rwanda (2010), 'Development of the telecom sector for the last three years', Rwanda Utilities Regulatory Agency (RURA).

Schechter, L. \& Yuskavage, A. (2011), 'Reciprocated versus unreciprocated sharing in social networks', SSRN eLibrary .

Sobel, J. (2005), 'Interdependent preferences and reciprocity', Journal of Economic Literature 43(2), 392-436. ArticleType: research-article / Full publication date: Jun., 2005 / Copyright 2005 American Economic Association. Suri, T., Jack, B. \& Stoker, T. (2012), 'Dcoumenting the birth of a fincancial economy', Proceedings of the National Academy of Sciences . 
Townsend, R. M. (1994), 'Risk and insurance in village India', Econometrica: Journal of the Econometric Society 62(3), 539-591.

Tukey, J. W. (1962), 'The future of data analysis', The Annals of Mathematical Statistics p. 167.

Udry, C. (1994), 'Risk and insurance in a rural credit market: An empirical investigation in northern nigeria', The Review of Economic Studies 61(3), 495-526.

Ureta, S. (2005), 'Variations on expenditure on communications in developing countries: A synthesis of the evidence from albania, mexico, nepal and south africa (2000-2003)', Diversifying Participation in Network Development .

USGS (2009), 'Magnitude 5.9 - lac kivu region, dem. rep. of the congo'.

*http://earthquake.usgs.gov/eqcenter/eqinthenews/2008/us2008mzam/

Wooldridge, J. M. (2005), 'Simple solutions to the initial conditions problem in dynamic, nonlinear panel data models with unobserved heterogeneity', Journal of Applied Econometrics 20(1), 39-54.

World Bank Group (2009), 'Remittance prices world wide', Available at http://remittanceprices.worldbank.org . 


\section{Tables}

Table 1: Alternative methods for transferring money in Rwanda, c. 2008

\begin{tabular}{|c|c|c|c|c|}
\hline Service & $\begin{array}{l}\text { Estimated Fees } \\
\text { (small transfers) }\end{array}$ & Availability & Approx. Usage & Source \\
\hline Mobile Airtime Transfer & Free & $\begin{array}{l}2 \text { million phones, } 700 \\
\text { agents }\end{array}$ & $20 \%-32 \%$ & (1) \\
\hline MoneyGram & $\begin{array}{l}7 \%-100 \% \\
(\$ 15 \text { minimum })\end{array}$ & 5 locations & $1 \%$ & (2) \\
\hline Western Union & $\begin{array}{l}10 \%-100 \% \\
\text { (\$10 minimum) }\end{array}$ & 50 locations & $3 \%-14 \%$ & (3) \\
\hline Post Office & $8 \%-50 \%$ & 19 branches & $1 \%$ & $(4)$ \\
\hline Commercial Bank & $6 \%-40 \%$ & $\begin{array}{l}\text { Only in urban, semi- } \\
\text { urban areas }\end{array}$ & $9 \%-10 \%$ & $(4)$ \\
\hline Public Bus & $6 \%-20 \%$ & Populous areas & $22 \%-28 \%$ & (5) \\
\hline
\end{tabular}

Notes: Data on fees and availability compiled in from the following sources: (1) Phone company records, (2) MoneyGram Website, (3) Western Union Website, (4) World Bank Group (2009), (5) Average of rates for Bus-Star and Scandinavian Transportation. Data on usage obtained from Blumenstock \& Eagle (2012), and based on 910 phone interview responses to the question, "What method do you use most frequently to send/receive money?" See Orozco (2009) and Kabbucho et al. (2003) for further quantitative estimates, or Collins et al. (2009) for a more general overview. 
Table 2: Summary statistics of mobile network data.

\begin{tabular}{|c|c|c|}
\hline & $\begin{array}{c}\text { All dates } \\
10 / 1 / 2006-7 / 1 / 2008\end{array}$ & $\begin{array}{l}\text { Earthquake window } \\
1 / 3 / 2008-3 / 3 / 2008\end{array}$ \\
\hline \multicolumn{3}{|l|}{ Panel A: Aggregate traffic } \\
\hline Number of Calls & $46,000,000,000$ & $868,786,684$ \\
\hline Number of interpersonal transfers & $9,202,954$ & 362,053 \\
\hline Number of unique users & $1,084,085$ & 119,745 \\
\hline Number of people who send airtime & 870,099 & 48,295 \\
\hline Number of people who receive airtime & 946,855 & 101,351 \\
\hline Number of people who both send and receive & 732,869 & 29,901 \\
\hline Number of unique dyads & 646,713 & 159,204 \\
\hline \multicolumn{3}{|l|}{ Panel B: Transaction and dyad statistics } \\
\hline Transactions per user (send+receive) & 16.98 & 6.05 \\
\hline Average distance per transaction $(\mathrm{km})$ & 10.28 & 8.16 \\
\hline Average transaction value (RWF) & 258.51 & 237.71 \\
\hline Average social proximity $S_{i j}$ & 34.73 & 26.18 \\
\hline \multicolumn{3}{|l|}{ Panel C: Individual characteristics (phone survey) } \\
\hline Average annual expenditures (RWF) & 948750 & - \\
\hline Percent male & $66 \%$ & - \\
\hline Average age & 32.03 & - \\
\hline Average household size & 5.87 & - \\
\hline
\end{tabular}


Table 3: Average Effect of the Earthquake on Transfers Received

\begin{tabular}{|c|c|c|c|c|}
\hline & $\begin{array}{c}(1) \\
\text { District }\end{array}$ & $\begin{array}{c}(2) \\
\text { Cell Tower }\end{array}$ & $\begin{array}{c}(3) \\
\text { User }\end{array}$ & $\begin{array}{c}(4) \\
\text { Dyad }\end{array}$ \\
\hline \multicolumn{5}{|c|}{ Panel A: Gross transfers received (total incoming) } \\
\hline Earthquake Shock & $\begin{array}{c}14,169^{* * *} \\
(1,951)\end{array}$ & $\begin{array}{c}2,832^{* * *} \\
(177)\end{array}$ & $\begin{array}{c}9.47^{* * *} \\
(0.74)\end{array}$ & $\begin{array}{c}8.14^{* * *} \\
(1.34)\end{array}$ \\
\hline NearEpicenter ${ }_{i t}$ & & & $\begin{array}{c}0.09 \\
(0.28)\end{array}$ & $\begin{array}{l}0.18^{*} \\
(0.11)\end{array}$ \\
\hline Unconditional Mean & 19,007 & 2,436 & 5.90 & 3.65 \\
\hline Unconditional Mean (affected area) & 6356 & 1245 & 3.8 & 3.2 \\
\hline \multicolumn{5}{|c|}{ Panel B: Net transfers received (incoming - outgoing) } \\
\hline Earthquake shock & $\begin{array}{c}12,823^{* * *} \\
(1,600)\end{array}$ & $\begin{array}{c}3,053^{* * *} \\
(116)\end{array}$ & $\begin{array}{c}10.00^{* * *} \\
(1.08)\end{array}$ & $\begin{array}{c}8.816^{* * *} \\
(1.65)\end{array}$ \\
\hline NearEpicenter ${ }_{i t}$ & & & $\begin{array}{c}0.60 \\
(0.46) \\
\end{array}$ & $\begin{array}{l}0.45^{+} \\
(0.24) \\
\end{array}$ \\
\hline Unconditional Mean & 0 & 0 & 0 & 0 \\
\hline Unconditional Mean (affected area) & 1,399 & 248 & 0.514 & 0.64 \\
\hline \multicolumn{5}{|l|}{ Panel C: Total cost of calls received } \\
\hline Earthquake Shock & $\begin{array}{c}2,501,220^{* *} \\
(886,529)\end{array}$ & $\begin{array}{c}565,330 * * \\
(168,349)\end{array}$ & $\begin{array}{c}247.95^{* * *} \\
(34.21)\end{array}$ & - \\
\hline NearEpicenter ${ }_{i t}$ & & & $\begin{array}{c}-22.17^{* * *} \\
(5.89) \\
\end{array}$ & - \\
\hline Unconditional Mean & $3,845,858$ & 425,740 & 162.91 & \\
\hline Unconditional Mean (affected area) & $1,843,455$ & 353,501 & 154.17 & \\
\hline Number of observations & 1,800 & 16,020 & $6,704,973^{\ddagger}$ & $10,032,721$ \\
\hline Day dummies & Yes & Yes & Yes & Yes \\
\hline Fixed effects & District & Tower & Individual & Dyad (Directed) \\
\hline
\end{tabular}

Notes: In Panels A and B, the dependent variable is the gross (Panel A) and net (Panel B) transfers received on a given day by a given district (column 1), cell tower (column 2), individual subscriber (column 3), or by an individual $i$ from a specific individual $j$ (column 4). In Panel C, the dependent variable is the total amount of money spent calling the district/tower/subscriber on each day. The Earthquake Shock variable takes the value one for regions/subscribers who were within $20 \mathrm{~km}$ of epicenter on the day of the earthquake; NearEpicenter it $_{\text {takes }}$ the value one for all observations where the subscriber is within $20 \mathrm{~km}$ of the epicenter (even when there is no earthquake). The unconditional mean reports the average of the dependent variable across the entire 2-month window (January 42008 - March 3 2008), for the country as a whole and for the region affected by the earthquake. $\ddagger$ Column (3) of Panel C includes all phone subscribers, not just individuals who had used the mobile money service, and includes 35,539,241 observations. We do not estimate column (4) of Panel C because the roughly 100 billion observations made the computation infeasible. Standard errors, clustered by district, reported in parentheses. ${ }^{*}$ significant at $p<.05 ;{ }^{* *} p<.01 ;{ }^{* * *} p<.001$. 
Table 4: Net transfers and wealth

\begin{tabular}{|c|c|c|c|}
\hline & (1) & $(2)$ & (3) \\
\hline & No Fixed Effects & Avg. Partial Effects & Fixed Effects \\
\hline \multirow[t]{3}{*}{ Earthquake Shock } & $10.206^{* * *}$ & & $9.794^{* * *}$ \\
\hline & $(4.56)$ & & $(4.33)$ \\
\hline & {$[0.000]$} & & {$[0.000]$} \\
\hline \multirow[t]{3}{*}{ Recipient Wealth $x_{i} *$ Shock } & $5.767^{*}$ & & $5.763^{*}$ \\
\hline & $(1.11)$ & & $(1.26)$ \\
\hline & {$[0.082]$} & & {$[0.082]$} \\
\hline \multirow[t]{3}{*}{ Sender Wealth $x_{j} *$ Shock } & $15.682^{*}$ & & $14.903^{*}$ \\
\hline & $(14.39)$ & & $(13.33)$ \\
\hline & {$[0.085]$} & & {$[0.076]$} \\
\hline \multirow[t]{3}{*}{ Social Proximity $S_{i j} *$ Shock } & 0.101 & & 0.110 \\
\hline & $(0.08)$ & & $(0.07)$ \\
\hline & {$[0.331]$} & & {$[0.268]$} \\
\hline \multirow[t]{3}{*}{ Recipient Wealth $\left(x_{i}\right)$} & $0.863^{* * *}$ & $0.976^{* * *}$ & \\
\hline & $(0.17)$ & $(0.09)$ & \\
\hline & {$[0.001]$} & {$[0.001]$} & \\
\hline \multirow[t]{3}{*}{ Sender Wealth $\left(x_{j}\right)$} & $1.641^{* * *}$ & $1.618^{* * *}$ & \\
\hline & $(0.11)$ & $(0.13)$ & \\
\hline & {$[0.000]$} & {$[0.000]$} & \\
\hline \multirow[t]{3}{*}{ Social Proximity $\left(S_{i j}\right)$} & $0.040^{* * *}$ & $0.040^{* * *}$ & \\
\hline & $(0.00)$ & $(0.00)$ & \\
\hline & {$[0.000]$} & {$[0.000]$} & \\
\hline Day dummies & Yes & Yes & Yes \\
\hline Fixed effects & None & None & Dyad (directed) \\
\hline Number of observations & 10032721 & 174799 & 10032721 \\
\hline Number of iterations (bootstrap) & 1000 & 1000 & 1000 \\
\hline
\end{tabular}

Notes: Outcome is $\tau_{i j t}$, the total airtime received by $i$ from $j$ on day $t$. Mean effects reported in Table 3 . Interaction terms $x_{i}, x_{j}$, and $s_{i j}$ are centered at the population mean. Standard errors, clustered by district, reported in parentheses as in other tables. To account for the fact that $x_{i}$ and $x_{j}$ are generated regressors, $P$-values, obtained from 1,000 simulations of bootstrapped estimation of the sequence of equations (6), (7), and (5), are reported in brackets. The p-value is the proportion of iterations where the estimated coefficient is found to have the opposite sign of the reported coefficient. Regressions include observations from the period January 4, 2009 through March 3, 2008. Wealth proxies $x_{i}$ and $x_{j}$ are computed using equations (6) and (7), as described in the text. $S_{i j}$ is measured by counting the number of phone calls between $i$ and $j$ in the last three months of 2007. All regressions include NearEpicenter it $_{\text {and }}$ anirwise interaction terms (e.g. $x_{i} *$ NearEpicenter $_{i t}$, $x_{i}{ }^{*}$ DayOfQuaket); these coefficients are omitted for clarity. ${ }^{* * *} \mathrm{p}<0.01,{ }^{* *} \mathrm{p}<0.05,{ }^{*} \mathrm{p}<0.10$. 
Table 5: Robustness check: Net transfers and wealth

\begin{tabular}{|c|c|c|c|c|}
\hline & $\begin{array}{c}(1) \\
\text { Original }\end{array}$ & $\begin{array}{c}(2) \\
\text { With Controls }\end{array}$ & $\begin{array}{c}\text { (3) } \\
\text { Airtime proxy } \\
\text { for wealth }\end{array}$ & $\begin{array}{l}\text { (4) } \\
\text { Net incoming calls } \\
\text { proxy for poverty }\end{array}$ \\
\hline Shock (recipient) & $\begin{array}{l}10.206^{*} \\
(4.557)\end{array}$ & $\begin{array}{l}9.803^{*} \\
(4.669)\end{array}$ & $\begin{array}{c}7.498^{* * *} \\
(0.353)\end{array}$ & $\begin{array}{c}7.925^{* * *} \\
(0.267)\end{array}$ \\
\hline Recipient Wealth $x_{i} *$ Shock & $\begin{array}{c}5.767^{* * *} \\
(1.113)\end{array}$ & $\begin{array}{l}8.764^{* *} \\
(2.512)\end{array}$ & & \\
\hline Sender Wealth $x_{j} *$ Shock & $\begin{array}{c}15.682 \\
(14.391)\end{array}$ & $\begin{array}{c}15.745 \\
(14.402)\end{array}$ & & \\
\hline Recipient Total Recharges * Shock & & & $\begin{array}{l}0.027^{* *} \\
(0.009)\end{array}$ & \\
\hline Sender Total Recharges $*$ Shock & & & $\begin{array}{l}0.046^{*} \\
(0.020)\end{array}$ & \\
\hline Recipient Net Incoming Calls * Shock & & & & $\begin{array}{r}-0.012^{+} \\
(0.007)\end{array}$ \\
\hline Sender Net Incoming Calls * Shock & & & & $\begin{array}{l}-0.025^{*} \\
(0.012)\end{array}$ \\
\hline Recipient Calling Activity * Shock & & $\begin{array}{c}-0.004^{+} \\
(0.002)\end{array}$ & & \\
\hline Recipient Calling Activity & & $\begin{array}{c}-0.001^{* * *} \\
(0.0001)\end{array}$ & & \\
\hline Social Proximity $S_{i j} *$ Shock & $\begin{array}{c}0.101 \\
(0.077)\end{array}$ & $\begin{array}{l}0.118^{+} \\
(0.067)\end{array}$ & $\begin{array}{r}-0.024^{+} \\
(0.013)\end{array}$ & $\begin{array}{c}0.195 \\
(0.131)\end{array}$ \\
\hline Day dummies & Yes & Yes & Yes & Yes \\
\hline Fixed effects & None & None & None & None \\
\hline
\end{tabular}

Notes: Outcome in all specifications is $\tau_{i j t}$, i.e. airtime received by $i$ from $j$ on day $t$. Column 1 is identical to Column 1 of Table 4, where standard errors are estimated without bootstrapping, for comparison with later columns. Uninteracted coefficients are omitted for clarity. Column 2 includes the total calls made by $i$ in 2007 as an additional control for the total calling activity of the recipient. In Column 3, the measure of wealth is the the total number of airtime recharges by $i$ in 2007, instead of the predicted expenditures variable used in Columns 1 and 2. Column 4 utilizes the net incoming calls in 2007 (calls received minus calls made) as a measure that is negatively correlated with wealth. Standard errors, clustered by district, reported in parentheses. ${ }^{* * *} \mathrm{p}<0.001,{ }^{* *} \mathrm{p}<0.01,{ }^{*} \mathrm{p}<0.05,+\mathrm{p}<0.10$. 
Table 6: Net transfers and Distance

\begin{tabular}{lccc}
\hline \hline & $(1)$ & $(2)$ & $(3)$ \\
& No Fixed Effects & Avg. Partial Effects & Fixed Effects \\
\hline Shock (recipient) & $4.191^{* *}$ & & $3.901^{* *}$ \\
& $(1.392)$ & & $(1.307)$ \\
Distance $D_{i j t} *$ Shock & $-0.117^{*}$ & & $-0.116^{*}$ \\
& $(0.052)$ & & $(0.051)$ \\
Social Proximity $S_{i j} *$ Shock & 0.188 & & 0.199 \\
& $(0.128)$ & & $(0.121)$ \\
Distance $D_{i j t}$ & $-0.013^{* *}$ & & $-0.021^{* * *}$ \\
& $(0.004)$ & & $(0.001)$ \\
Social Proximity $S_{i j}$ & $0.050^{* * *}$ & $0.050^{* * *}$ & \\
& $(0.003)$ & $(0.002)$ & yes \\
\hline Day dummies & yes & yes & Dyad (directed) \\
Fixed effects & None & None & 9915422 \\
Number of observations & 9915422 & 193256 & \\
\hline \hline
\end{tabular}

Notes: Outcome is $\tau_{i j t}$, the total airtime received by $i$ from $j$ on day $t$. Mean effects reported in Table 3. Regressions include observations from the period January 4, 2008 through March 3, 2008. $D_{i j t}$ measures the distance between $i$ and $j$ in kilometers on day $t$, using the locational inference algorithm described in Section 4.1. $S_{i j}$ is measured by counting the number of phone calls between $i$ and $j$ in the last three months of 2007. All regressions include NearEpicenter it $_{\text {E }}$ and pairwise interaction terms (e.g. $D_{i j t} *$ NearEpicenter $i t, D_{i j t}{ }^{*}$ DayOfQuake $_{t}$ ); these coefficients are omitted for clarity. Standard errors, clustered by district, reported in parentheses. ${ }^{* * *} \mathrm{p}<0.001,{ }^{* *} \mathrm{p}<0.01,{ }^{*} \mathrm{p}<0.05,+\mathrm{p}<0.10$. 
Table 7: Net transfers and History-Dependence

\begin{tabular}{lccc}
\hline \hline & $(1)$ & $(2)$ & $(3)$ \\
& No Fixed Effects & Avg. Partial Effects & Fixed Effects \\
\hline Earthquake Shock $($ to $i)$ & $8.866^{* * *}$ & & $8.403^{* * *}$ \\
& $(2.106)$ & & $(1.873)$ \\
Net Bal. Outgoing Airtime $T_{j i t}^{\text {net } * \text { Shock }}$ & $0.006^{*}$ & & $0.006^{*}$ \\
& $(0.003)$ & & $(0.003)$ \\
Social Proximity $S_{i j}^{*}$ Shock & 0.192 & & 0.200 \\
& $(0.139)$ & $-0.011^{* * *}$ & $-0.010^{* * *}$ \\
Net Balance of Outgoing Airtime $\left(T_{j i t}^{n e t}\right)$ & $-0.001^{* * *}$ & $(0.0002)$ & $(0.0006)$ \\
& $(0.0002)$ & $0.051^{* * *}$ & \\
Social Proximity $\left(S_{i j}\right)$ & $0.051^{* * *}$ & $(0.003)$ & \\
& $(0.003)$ & Yes & Yes \\
\hline Day dummies & Yes & None & Dyad (directed) \\
Fixed effects & None & 174799 & 10032721 \\
Number of observations & 10032721 & \\
\hline \hline
\end{tabular}

Notes: Outcome is $\tau_{i j t}$, the total airtime received by $i$ from $j$ on day $t$. Mean effects reported in Table 3. Regressions include observations from the period January 4, 2009 through March 3, 2008. The net balance of outgoing airtime $T_{j i t}^{n e t}$ is measured as the total volume of airtime sent from $i$ to $j$ minus the total volume of airtime received by $i$ from $j$ prior to $t . S_{i j}$ is measured by counting the number of phone calls between $i$ and $j$ in the last three months of 2007. All regressions include NearEpicenter Et $_{\text {it }}$ and pairwise interaction terms (e.g. $T_{j i t}^{\text {net }} *$ NearEpicenter $_{i t}$, $T_{j i t}^{\text {net }}{ }^{\text {DayOfQuake }}$ ); these coefficients are omitted for clarity. Standard errors, clustered by district, reported in parentheses. ${ }^{* * *} \mathrm{p}<0.001,{ }^{* *} \mathrm{p}<0.01,{ }^{*} \mathrm{p}<0.05,+\mathrm{p}<0.10$.

Table 8: Predicted and actual comparative statics for charity and reciprocity

(1)

$(2)$
(3)
(5)

Predicted

\begin{tabular}{clccc} 
Partial & Interpretation & Charity & Reciprocity & Observed \\
\hline$\partial \tau_{i j t} / \partial x_{i}$ & Wealth of $i$ (recipient) & Negative & - & Positive \\
$\partial \tau_{i j t} / \partial x_{j}$ & Wealth of $j$ (sender) & Positive & - & - \\
$\partial \tau_{i j t} / \partial S_{i j}$ & Social connectedness of $i$ and $j$ (recipient) & Positive & Positive & Positive \\
$\partial \tau_{i j t} / \partial D_{i j t}$ & Geographic distance between $i$ and $j$ at time $t$ & - & Negative & Negative \\
$\partial \tau_{i j t} / \partial T_{i j t}^{\text {net }}$ & Net balance of transfers from $j$ to $i$ & (Positive) & Negative & Negative \\
\hline \hline
\end{tabular}

Notes: Summary of predictions of stylized models of charity and reciprocity presented in Section A, and results described in Section 5. Parentheses indicate weak predictions. 
Table 9: Placebo Tests - Region

\begin{tabular}{|c|c|c|c|c|c|}
\hline & (1) & $(2)$ & (3) & (4) & (5) \\
\hline & 3 month early & 2 months early & 1 month early & Actual Quake & 1 month late \\
\hline \multirow[t]{2}{*}{ placebo } & -2056.429 & 312.790 & 798.729 & $13326.179^{* * *}$ & -1089.114 \\
\hline & $(2471.31)$ & $(1662.70)$ & $(869.67)$ & $(1337.56)$ & $(1651.68)$ \\
\hline \multirow[t]{2}{*}{ placebo_lag1 } & -4438.345 & 902.978 & 196.765 & -992.363 & -133.638 \\
\hline & $(3292.15)$ & $(1259.07)$ & $(1342.27)$ & $(1215.16)$ & $(3688.71)$ \\
\hline \multirow[t]{2}{*}{ placebo_lag2 } & -1628.159 & 2961.500 & 80.133 & 1495.821 & -7377.086 \\
\hline & $(1967.20)$ & $(2775.80)$ & $(2661.46)$ & $(2678.79)$ & $(5687.47)$ \\
\hline \multirow[t]{2}{*}{ placebo_lag3 } & 1370.931 & 1437.113 & 2430.937 & 789.919 & -1949.726 \\
\hline & $(1115.80)$ & $(1711.70)$ & $(3958.41)$ & $(3092.60)$ & (1931.41) \\
\hline \multirow[t]{2}{*}{ placebo_lag4 } & -2393.805 & -1599.899 & -33.634 & -207.856 & -3785.758 \\
\hline & $(2354.55)$ & $(2108.57)$ & $(1691.97)$ & $(1407.14)$ & $(3793.98)$ \\
\hline \multirow[t]{2}{*}{ placebo_lag5 } & 1154.533 & 1153.144 & 611.661 & -44.916 & -3324.983 \\
\hline & $(1127.09)$ & $(1005.35)$ & $(1514.86)$ & $(2914.84)$ & $(2332.18)$ \\
\hline \multirow[t]{2}{*}{ placebo_lag6 } & $1482.134^{+}$ & -1509.240 & -324.826 & -2662.037 & -53.801 \\
\hline & $(748.31)$ & (1069.94) & $(1044.35)$ & $(3156.40)$ & $(2037.16)$ \\
\hline \multirow[t]{2}{*}{ placebo_lag7 } & $1371.100^{+}$ & 963.621 & $1542.388^{+}$ & -401.769 & 1764.977 \\
\hline & $(709.54)$ & $(735.43)$ & $(807.87)$ & $(810.92)$ & $(2372.48)$ \\
\hline \multirow[t]{2}{*}{ Total calls } & 0.010 & 0.010 & 0.010 & 0.010 & 0.010 \\
\hline & $(0.01)$ & $(0.01)$ & $(0.01)$ & $(0.01)$ & $(0.01)$ \\
\hline \multirow[t]{2}{*}{ Outgoing transfers } & $0.878^{* * *}$ & $0.878^{* * *}$ & $0.878^{* * *}$ & $0.878^{* * *}$ & $0.878^{* * *}$ \\
\hline & $(0.02)$ & $(0.02)$ & $(0.02)$ & $(0.02)$ & $(0.02)$ \\
\hline Constant & $2431.066^{* * *}$ & $2430.638^{* * *}$ & $2430.741^{* * *}$ & $2431.985^{* * *}$ & $2432.176^{* * *}$ \\
\hline $\mathrm{N}$ & 18990 & 18990 & 18990 & 18990 & 18990 \\
\hline
\end{tabular}

Outcome: Value of incoming airtime sent to people in district (in RWF; US $\$ 1=500 \mathrm{RWF}$ ). Heteroskedasticity-robust SE's in parentheses (clustered at district level). 
Table 10: Lagged effects of the earthquake on transfers and calls received.

\begin{tabular}{|c|c|c|c|}
\hline & $\begin{array}{c}(1) \\
\text { Transfers Received }\end{array}$ & $\begin{array}{c}\text { (2) } \\
\text { Calls Received }\end{array}$ & $\begin{array}{c}(3) \\
\text { Int'l Calls Received }\end{array}$ \\
\hline Shock & $\begin{array}{c}13599.987^{* * *} \\
(1051.04)\end{array}$ & $\begin{array}{c}14206.422^{* * *} \\
(3762.18)\end{array}$ & $\begin{array}{c}141.634^{*} \\
(57.11)\end{array}$ \\
\hline shock_lag1 & $\begin{array}{l}-1200.544 \\
(1129.42)\end{array}$ & $\begin{array}{c}4590.319^{* * *} \\
(507.72)\end{array}$ & $\begin{array}{l}124.644 \\
(77.40)\end{array}$ \\
\hline shock_lag2 & $\begin{array}{l}1444.242 \\
(2848.82)\end{array}$ & $\begin{array}{l}1635.936 \\
(1035.70)\end{array}$ & $\begin{array}{l}60.666 \\
(50.72)\end{array}$ \\
\hline shock_lag3 & $\begin{array}{c}595.617 \\
(3277.26)\end{array}$ & $\begin{array}{c}1293.153^{* * *} \\
(299.40)\end{array}$ & $\begin{array}{l}45.264 \\
(33.24)\end{array}$ \\
\hline shock_lag4 & $\begin{array}{l}-428.624 \\
(1379.12)\end{array}$ & $\begin{array}{l}547.864^{*} \\
(206.22)\end{array}$ & $\begin{array}{c}-30.650^{+} \\
(17.38)\end{array}$ \\
\hline shock_lag5 & $\begin{array}{l}-189.757 \\
(3162.41)\end{array}$ & $\begin{array}{c}1066.467^{* * *} \\
(228.90)\end{array}$ & $\begin{array}{c}-68.270^{*} \\
(29.36)\end{array}$ \\
\hline shock_lead1 & $\begin{array}{c}567.236 \\
(1886.17)\end{array}$ & $\begin{array}{l}224.344 \\
(322.57)\end{array}$ & $\begin{array}{l}32.700 \\
(25.67)\end{array}$ \\
\hline shock_lead2 & $\begin{array}{l}1198.341 \\
(1119.03)\end{array}$ & $\begin{array}{l}215.258 \\
(388.01)\end{array}$ & $\begin{array}{l}38.347 \\
(44.66)\end{array}$ \\
\hline shock_lead3 & $\begin{array}{l}-2727.993 \\
(1802.28)\end{array}$ & $\begin{array}{l}-77.107 \\
(207.44)\end{array}$ & $\begin{array}{r}-26.907 \\
(60.26)\end{array}$ \\
\hline shock_lead4 & $\begin{array}{l}-117.459 \\
(2098.99)\end{array}$ & $\begin{array}{c}-489.221^{*} \\
(194.31)\end{array}$ & $\begin{array}{l}-17.748 \\
(50.78)\end{array}$ \\
\hline shock_lead5 & $\begin{array}{l}6681.922 \\
(6234.18)\end{array}$ & $\begin{array}{l}-83.052 \\
(236.95)\end{array}$ & $\begin{array}{l}17.834 \\
(58.40)\end{array}$ \\
\hline Outgoing transfers & $\begin{array}{c}0.884^{* * *} \\
(0.02)\end{array}$ & & \\
\hline Outgoing calls & & $\begin{array}{c}0.969^{* * *} \\
(0.00)\end{array}$ & \\
\hline Outgoing international calls & & & $\begin{array}{c}0.953^{* * *} \\
(0.02)\end{array}$ \\
\hline $\mathrm{N}$ & 19200 & 19200 & 19200 \\
\hline
\end{tabular}


Table 11: Effect of flood on transfers

\begin{tabular}{|c|c|c|c|c|}
\hline & $\begin{array}{c}(1) \\
\text { Pooled OLS }\end{array}$ & $\begin{array}{c}(2) \\
\text { OLS controls }\end{array}$ & $\begin{array}{l}(3) \\
\text { tower FE }\end{array}$ & $\begin{array}{c}(4) \\
\text { tower/Time FE }\end{array}$ \\
\hline Flood shock & $\begin{array}{c}1455.411^{+} \\
(770.90)\end{array}$ & $\begin{array}{l}772.401^{*} \\
(290.32)\end{array}$ & $\begin{array}{l}818.131^{*} \\
(312.69)\end{array}$ & $\begin{array}{l}849.166^{*} \\
(344.89)\end{array}$ \\
\hline DaysOf Flood $_{t}$ & $\begin{array}{c}781.550^{* * *} \\
(166.88)\end{array}$ & $\begin{array}{c}361.117^{* * *} \\
(78.07)\end{array}$ & $\begin{array}{c}366.729^{* * *} \\
(72.11)\end{array}$ & \\
\hline NearFloodRegion $_{i t}$ & $\begin{array}{l}264.965 \\
(919.89)\end{array}$ & $\begin{array}{c}173.885^{* *} \\
(57.73)\end{array}$ & & \\
\hline Outgoing transfers & & $\begin{array}{c}0.679^{* * *} \\
(0.03)\end{array}$ & $\begin{array}{c}0.640^{* * *} \\
(0.03)\end{array}$ & $\begin{array}{c}0.599^{* * *} \\
(0.03)\end{array}$ \\
\hline Total calls & & $\begin{array}{c}0.088^{* * *} \\
(0.00)\end{array}$ & $\begin{array}{c}0.070^{* * *} \\
(0.01)\end{array}$ & $\begin{array}{c}0.084^{* * *} \\
(0.01)\end{array}$ \\
\hline Tower Fixed Effects & No & No & Yes & Yes \\
\hline Date Fixed Effects & No & No & No & Yes \\
\hline$R^{2}$ & 0.000 & 0.785 & 0.804 & 0.811 \\
\hline$N$ & 170880 & 170880 & 170880 & 170880 \\
\hline
\end{tabular}

"In flood region" defined as towers in the two districts affected by the flood. "Days of flood" are 9/12/07 - 9/18/07. 


\section{Appendix Tables}

Appendix Table 1: Sensitivity of estimation to functional form assumptions

\begin{tabular}{lcccc}
\hline \hline & $(1)$ & $(2)$ & $(3)$ & $(4)$ \\
& Pooled OLS & OLS w/Controls & Region FE & Region \& Day FE \\
\hline Earthquake Shock & $2856.952^{* * *}$ & $2193.288^{* * *}$ & $2355.990^{* * *}$ & $2209.329^{* * *}$ \\
& $(416.57)$ & $(266.57)$ & $(176.02)$ & $(236.19)$ \\
DayOfQuake & $-717.340^{* *}$ & $-749.883^{* * *}$ & $-679.739^{* * *}$ & \\
NearEpicenter $i t$ & $(211.62)$ & $(98.79)$ & $(109.77)$ & \\
& $-1342.708^{*}$ & $-124.474^{* * *}$ & & \\
Outgoing transfers & $(546.53)$ & $(27.93)$ & & $0.599^{* * *}$ \\
& & $0.679^{* * *}$ & $0.640^{* * *}$ & $(0.03)$ \\
Total call volume & & $(0.03)$ & $(0.03)$ & $0.084^{* * *}$ \\
& & $0.088^{* * *}$ & $0.070^{* * *}$ & $(0.01)$ \\
\hline Tower Fixed Effects & No & $(0.00)$ & $(0.01)$ & Yes \\
Date Fixed Effects & No & No & Yes & No \\
$R^{2}$ & 0.001 & 0.785 & 0.804 & 0.811 \\
$N$ & 170880 & 170880 & 170880 & 170880 \\
\hline
\end{tabular}

Notes: Outcome is the total amount transferred into a tower on a single day. NearEpicenter defined as those towers within 20 miles of the earthquake epicenter. Columns 2-4 include controls for overall network activity at the tower-day level. Column 3 includes tower-level fixed effects. Column 4 includes daily fixed effects. Estimates made using data from October 1, 2006 through July 1, 2008. Heteroskedasticity-robust SE's in parentheses (clustered at district level). * $\mathrm{p}<0.05$, ** $\mathrm{p}<0.01, * * * \mathrm{p}<0.001$. 
Appendix Table 2: Sensitivity to outliers

(1)

(2)

(3)

(4)

(5)

\begin{tabular}{lccccc} 
& Original & Binary & Trimmed & Winsorised & Inv. hyperbolic sine \\
\hline Earthquake Shock & $8.136^{* * *}$ & $0.005^{* * *}$ & $4.275^{* * *}$ & $0.100^{* * *}$ & $0.050^{* * *}$ \\
& $(1.34)$ & $(0.00)$ & $(0.96)$ & $(0.01)$ & $(0.00)$ \\
Day of earthquake dummy & $-3.253^{* * *}$ & $-0.012^{* * *}$ & $-2.102^{* * *}$ & $-0.130^{* * *}$ & $-0.052^{* * *}$ \\
& $(0.53)$ & $(0.00)$ & $(0.21)$ & $(0.01)$ & $(0.00)$ \\
Recipient near epicenter & $0.229^{*}$ & $-0.005^{* * *}$ & -0.074 & $-0.042^{* * *}$ & $-0.015^{* * *}$ \\
& $(0.11)$ & $(0.00)$ & $(0.13)$ & $(0.01)$ & $(0.00)$ \\
\hline Day fixed effects & Yes & Yes & Yes & Yes & Yes \\
Fixed effects & Dyad & Dyad & Dyad & Dyad & Dyad \\
\hline$N$ & 10032721 & 10032721 & 10031628 & 10032721 & 10032721 \\
\hline \hline
\end{tabular}

Notes: Specifications are similar to that used to produce column (4) of Table 3, with different transformations of the dependent variable to reduce the influence of the long right tail of the dependent variable. Column (1) contains the original result for reference. Column (2) uses a binary dependent variable that is positive when recipient receives a non-zero transfer. Column (3) removes outliers by dropping values whether the transfer is greater than $2500 \mathrm{RWF}$, which is the 99 th percentile of non-zero transfers received. Column (4) winsorizes the dependent variable at the 99th percentile. Column (5) applies an inverse hyperbolic sine transformation to the dependent variable. ${ }^{*} \mathrm{p}<0.05, * * \mathrm{p}<0.01, * * * \mathrm{p}<0.001$.

Appendix Table 3: Sensitivity of standard errors in dyadic results

\begin{tabular}{lcccc}
\hline \hline & $(1)$ & $(2)$ & $(3)$ & $(4)$ \\
Clustering & $j$ 's District & $j$ 's Tower & $j$ & Day $t$ \\
\hline Earthquake Shock & $9.794^{* *}$ & $9.792^{* * *}$ & $9.892^{* * *}$ & $9.892^{* * *}$ \\
& $(4.33)$ & $(3.51)$ & $(3.77)$ & $(0.31)$ \\
$x_{i} *$ Shock & $5.763^{* * *}$ & $5.770^{* *}$ & $6.011^{*}$ & $6.011^{* * *}$ \\
& $(1.26)$ & $(2.89)$ & $(3.39)$ & $(0.40)$ \\
$x_{j} *$ Shock & 14.903 & 14.833 & 14.684 & $14.684^{* * *}$ \\
& $(13.33)$ & $(12.12)$ & $(11.86)$ & $(0.97)$ \\
$S_{i j} *$ Shock & 0.110 & 0.111 & 0.110 & $0.110^{* * *}$ \\
& $(0.07)$ & $(0.12)$ & $(0.15)$ & $(0.01)$ \\
\hline Day fixed effects & Yes & Yes & Yes & Yes \\
Fixed effects & Dyad & Dyad & Dyad & Dyad \\
\hline N & $10,032,721$ & 10,032,721 & $10,251,136$ & $10,251,136$ \\
\hline \hline
\end{tabular}


Appendix Table 4: Net transfers and wealth (Robustness to sender FE)

\begin{tabular}{|c|c|}
\hline Earthquake Shock & $\begin{array}{c}9.727^{*} \\
(4.36)\end{array}$ \\
\hline Recipient Wealth $x_{i} *$ Shock & $\begin{array}{c}5.702^{* * *} \\
(1.31)\end{array}$ \\
\hline Sender Wealth $\left(x_{j}\right) *$ Shock & $\begin{array}{c}15.299 \\
(13.93)\end{array}$ \\
\hline Social Proximity $S_{i j} *$ Shock & $\begin{array}{l}0.102 \\
(0.07)\end{array}$ \\
\hline Recipient Wealth $\left(x_{i}\right)$ & $\begin{array}{c}0.847^{* * *} \\
(0.17)\end{array}$ \\
\hline Social Proximity $\left(S_{i j}\right)$ & $\begin{array}{c}0.069 * * * \\
(0.00)\end{array}$ \\
\hline Day dummies & Yes \\
\hline Fixed effects & Sender $j$ \\
\hline Number of observations & 10032721 \\
\hline \multicolumn{2}{|c|}{$\begin{array}{l}\text { Notes: Outcome is } \tau_{i j t} \text {, the total airtime received by } i \text { from } j \text { on day } t \text {. Re- } \\
\text { gressions include observations from the period January } 4,2008 \text { through March } \\
3,2008 \text {. Wealth proxies } x_{i} \text { and } x_{j} \text { are computed using equations }(6) \text { and }(7) \text {, } \\
\text { as described in the text. } S_{i j} \text { is measured by counting the number of phone } \\
\text { calls between } i \text { and } j \text { in the last three months of } 2007 \text {. All regressions include } \\
\text { NearEpicenter } i \text { and pairwise interaction terms (e.g. } x_{i} * \text { NearEpicenter }{ }_{i t} \text {, } \\
\left.x_{i}{ }^{*} \text { DayOfQuake }\right)_{t} \text {; these coefficients are omitted for clarity. Standard er- } \\
\text { rors, clustered by district, reported in parentheses. }{ }^{* * *} \mathrm{p}<0.001,{ }^{* *} \mathrm{p}<0.01,{ }^{*} \\
\mathrm{p}<0.05,+\mathrm{p}<0.10 \text {. }\end{array}$} \\
\hline
\end{tabular}


Appendix Table 5: Network Flow as Social Proximity

\begin{tabular}{lccc}
\hline \hline & $(1)$ & $(2)$ & $(3)$ \\
& No Fixed Effects & Avg. Partial Effects & Fixed Effects \\
\hline Shock (recipient) & $8.935^{*}$ & & $8.480+$ \\
& $(4.33)$ & & $(4.16)$ \\
Recipient Wealth $x_{i}{ }^{*}$ Shock & $6.791^{* * *}$ & & $6.806^{* * *}$ \\
& $(0.75)$ & & $(0.95)$ \\
Sender Wealth $x_{j}{ }^{*}$ Shock & 16.501 & & 15.766 \\
& $(14.74)$ & & -0.846 \\
Network Flow $S_{i j}{ }^{*}$ Shock & -0.918 & & $(0.51)$ \\
& $(0.62)$ & $1.266^{* * *}$ & \\
Recipient Wealth $\left(x_{i}\right)$ & $1.146^{* * *}$ & $(0.10)$ & \\
& $(0.17)$ & $1.892^{* * *}$ & \\
Sender Wealth $\left(x_{j}\right)$ & $1.915^{* * *}$ & $(0.14)$ & \\
Network Flow $\left(S_{i j}\right)$ & $(0.12)$ & $0.054^{* * *}$ & \\
Day dummies & 0.048 & $(0.02)$ & Yes \\
Fixed effects & $(0.03)$ & Yes & None \\
Number of observations & Yes & 10032721 & 10032721 \\
\hline \hline
\end{tabular}

Notes: Outcome is $\tau_{i j t}$, i.e. airtime received by $i$ from $j$ on day $t . x_{i}$ and $x_{j}$ are estimated using equations (6) and (7), described in text. $S_{i j}$ measures the number of distinct paths between $i$ and $j$, where a path is defined by the call graph. All regressions include NearEpicenter $i$ and pairwise interaction terms (e.g. $x_{i}{ }^{*}$ NearEpicenter $i, x_{i}{ }^{*}$ DayOfQuake $e_{t}$ ), but results are omitted for clarity. Standard errors, clustered by district, reported in parentheses. ${ }^{* * *} \mathrm{p}<0.01,{ }^{* *} \mathrm{p}<0.05,{ }^{*} \mathrm{p}<0.1$.

Appendix Table 6: Regression of Expenditures on Asset Ownership

\begin{tabular}{l|cc|cc}
\hline \hline Outcome & \multicolumn{2}{|c|}{$\log$ (Expenditures) } & \multicolumn{2}{c}{ Expenditures } \\
& $\beta^{a}$ & (S.E.) & $\beta^{a}$ & (S.E.) \\
\hline Radio & 0.18 & $(0.02)$ & 40090 & $(13007)$ \\
Television & 1.14 & $(0.01)$ & 2130434 & $(44048)$ \\
Bed & 0.24 & $(0.04)$ & 187061 & $(8266)$ \\
Table & 0.13 & $(0.01)$ & 57601 & $(9109)$ \\
Car/Truck & 0.24 & $(0.01)$ & 1695284 & $(57718)$ \\
Motorcycle & 0.65 & $(0.04)$ & 8229976 & $(197091)$ \\
Bicycle & 0.22 & $(0.11)$ & 138186 & $(20359)$ \\
HH Size & 0.09 & $(0.02)$ & 56168 & $(3198)$ \\
\hline$R^{2}$ & \multicolumn{2}{|c|}{0.62} & \multicolumn{2}{|c}{0.75} \\
$N$ & 6900 & \multicolumn{2}{|c}{6900} \\
\hline \hline
\end{tabular}

Notes: Standard errors reported in parentheses. 
Appendix Table 7: Summary statistics of phone use as computed from transaction logs

\begin{tabular}{lc}
\hline \hline & Average \\
\hline Panel A: Domestic and & International Calls \\
Activation date & $1 / 12 / 08$ \\
Days of activity & 770.3 \\
Avg. call length & 31.7 \\
Calls per day & 6.25 \\
Net calls per day (out-in) & 0.087 \\
Int'l calls per day & 0.084 \\
Net int'l calls (out-in) & -0.014 \\
\hline Panel B: Social Network Structure \\
Degree & 734 \\
In-degree & 488.2 \\
Out-degree & 433 \\
Daily degree & 3.78 \\
Net daily degree (out-in) & 0.00027 \\
Clustering & 0.063 \\
Betweenness & 2.72 \\
\hline Panel C: Other Behaviors & \\
Credit used per day & 163.5 \\
Max. recharge value & 2756.3 \\
Avg. districts per day & 1.36 \\
Avg. districts contacted & 1.21 \\
$N$ & 901 \\
\hline \hline
\end{tabular}

Notes: Mean values reported, weighted by sampling strata to produce averages representative of entire phone population. 
Appendix Table 8: Regression of predicted expenditures on phone use

\begin{tabular}{|c|c|c|}
\hline & Coefficient & (S. E.) \\
\hline Num days phone was used & 1078.9 & $(0.94)$ \\
\hline Days $^{2}$ & 0.511 & $(0.66)$ \\
\hline Number of recharges & -2178.4 & $(-0.72)$ \\
\hline Recharges $^{2}$ & -5.063 & $(-1.75)$ \\
\hline Total calls made & 42.55 & $(0.08)$ \\
\hline Calls made $^{2}$ & 0.00446 & $(0.10)$ \\
\hline Total calls received & -1053.8 & $(-1.56)$ \\
\hline Calls received $^{2}$ & 0.0562 & $(0.82)$ \\
\hline Duration (outgoing) & $27.98^{* * *}$ & $(3.46)$ \\
\hline Duration (outgoing) ${ }^{2}$ & $-0.0000242^{* *}$ & $(-2.59)$ \\
\hline Duration (incoming) & $33.19^{* *}$ & $(2.74)$ \\
\hline Duration (incoming) $^{2}$ & -0.0000354 & $(-1.29)$ \\
\hline International calls made & -7853.7 & $(-0.72)$ \\
\hline International calls made ${ }^{2}$ & 43.95 & $(1.44)$ \\
\hline International calls received & -7191.3 & $(-0.70)$ \\
\hline International calls received $^{2}$ & 27.29 & $(1.06)$ \\
\hline Degree (outgoing) & -2607.8 & $(-0.29)$ \\
\hline Degree (outgoing) ${ }^{2}$ & 14.01 & $(1.09)$ \\
\hline Degree (incoming) & -7925.6 & $(-0.71)$ \\
\hline Degree (incoming) ${ }^{2}$ & 9.917 & $(0.85)$ \\
\hline Int'l Degree & $336694.7^{* *}$ & $(3.06)$ \\
\hline Int'l degree ${ }^{2}$ & -2.991 & $(-0.00)$ \\
\hline Districts called & -82424.9 & $(-0.78)$ \\
\hline Districts called $^{2}$ & -1440.6 & $(-0.55)$ \\
\hline Districts received & $-317282.5^{* *}$ & $(-3.06)$ \\
\hline Districts received $^{2}$ & -86.57 & $(-0.07)$ \\
\hline$R^{2}$ & \multicolumn{2}{|c|}{0.392} \\
\hline$N$ & \multicolumn{2}{|c|}{901} \\
\hline
\end{tabular}

Outcome is predicted expenditures $\widehat{Y_{i d}}$, in RWF. Standard errors in parentheses. Regression includes district fixed effects. * significant at $p<.05 ;{ }^{* *} p<.01 ;^{* * *} p<.001$. 


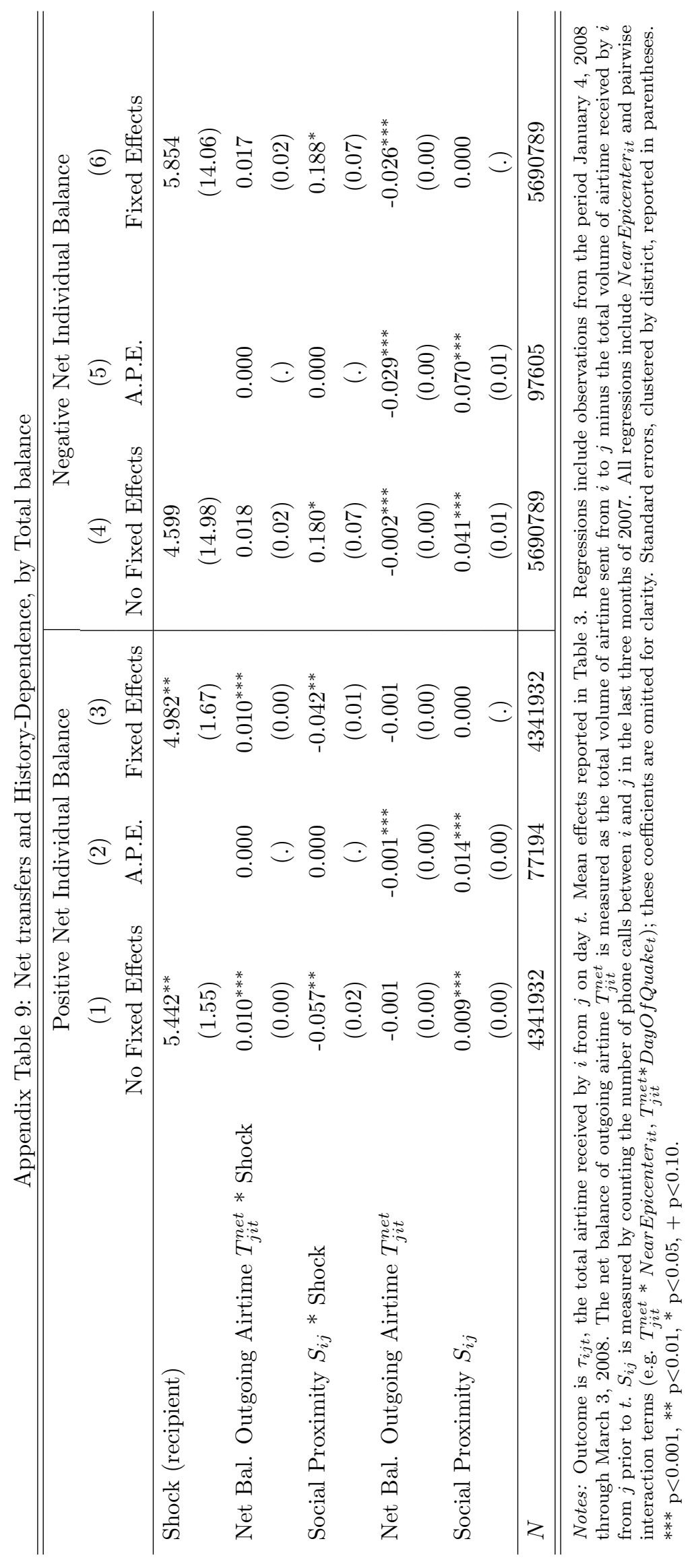


Appendix Table 10: Full interaction specification

\begin{tabular}{lccc}
\hline \hline & $(1)$ & $(2)$ & $(3)$ \\
& No F.E. & A.P.E. & Fixed Effects \\
\hline Shock (recipient) & $7.261^{+}$ & & $6.965^{*}$ \\
& $(3.59)$ & & $(3.38)$ \\
Recipient Wealth $x_{i} *$ Shock & $6.425^{* * *}$ & & $6.176^{* * *}$ \\
& $(0.23)$ & & $(0.33)$ \\
Sender Wealth $x_{j} *$ Shock & 15.238 & & 14.662 \\
& $(13.21)$ & & $(12.14)$ \\
Distance $D_{i j t} *$ Shock & $0.070^{+}$ & & $0.069^{+}$ \\
& $(0.04)$ & & $(0.04)$ \\
Net Bal. Outgoing Airtime $T_{j i t}^{\text {net }} *$ Shock & 0.006 & & 0.006 \\
& $(0.00)$ & & $(0.00)$ \\
Social Proximity $S_{i j} *$ Shock & 0.112 & & 0.127 \\
& $(0.10)$ & & $(0.09)$ \\
Recipient Wealth $x_{i}$ & $1.019^{* * *}$ & $0.935^{* * *}$ & \\
Sender Wealth $x_{j}$ & $(0.16)$ & $(0.11)$ & \\
Distance $D_{i j t}$ & $1.553^{* * *}$ & $1.809^{* * *}$ & \\
Net Bal. Outgoing Airtime $T_{j i t}^{n e t}$ & $(0.11)$ & $(0.10)$ & $-0.021^{* * *}$ \\
& $-0.013^{* * *}$ & $0.004^{* *}$ & $(0.00)$ \\
Social Proximity $S_{i j}$ & $(0.00)$ & $(0.00)$ & $-0.010^{* * *}$ \\
& $-0.001^{* * *}$ & $-0.011^{* * *}$ & $(0.00)$ \\
$N$ & $(0.00)$ & $(0.00)$ & \\
\hline \hline
\end{tabular}

Notes: Outcome is $\tau_{i j t}$, the total airtime received by $i$ from $j$ on day $t$. Regressions include observations from the period January 4, 2008 through March 3, 2008. Wealth proxies $x_{i}$ and $x_{j}$ are computed using equations (6) and (7), as described in the text. $D_{i j t}$ measures the distance between $i$ and $j$ in kilometers on day $t$, using the locational inference algorithm described in Section 4.1. The net balance of outgoing airtime $T_{j i t}^{\text {net }}$ is measured as the total volume of airtime sent from $i$ to $j$ minus the total volume of airtime received by $i$ from $j$ prior to $t . S_{i j}$ is measured by counting the number of phone calls between $i$ and $j$ in the last three months of 2007. All regressions include NearEpicenter ${ }_{i t}$ and pairwise interaction terms (e.g. $T_{j i t}^{\text {net }} *$ NearEpicenter $i t$, $T_{\text {jit }}^{\text {net } * \text { DayOfQuake }}$ ); these coefficients are omitted for clarity. Standard errors, clustered by district, reported in parentheses. ${ }^{* * *} \mathrm{p}<0.001,{ }^{* *} \mathrm{p}<0.01,{ }^{*} \mathrm{p}<0.05,+\mathrm{p}<0.10$. 


\section{Figures}

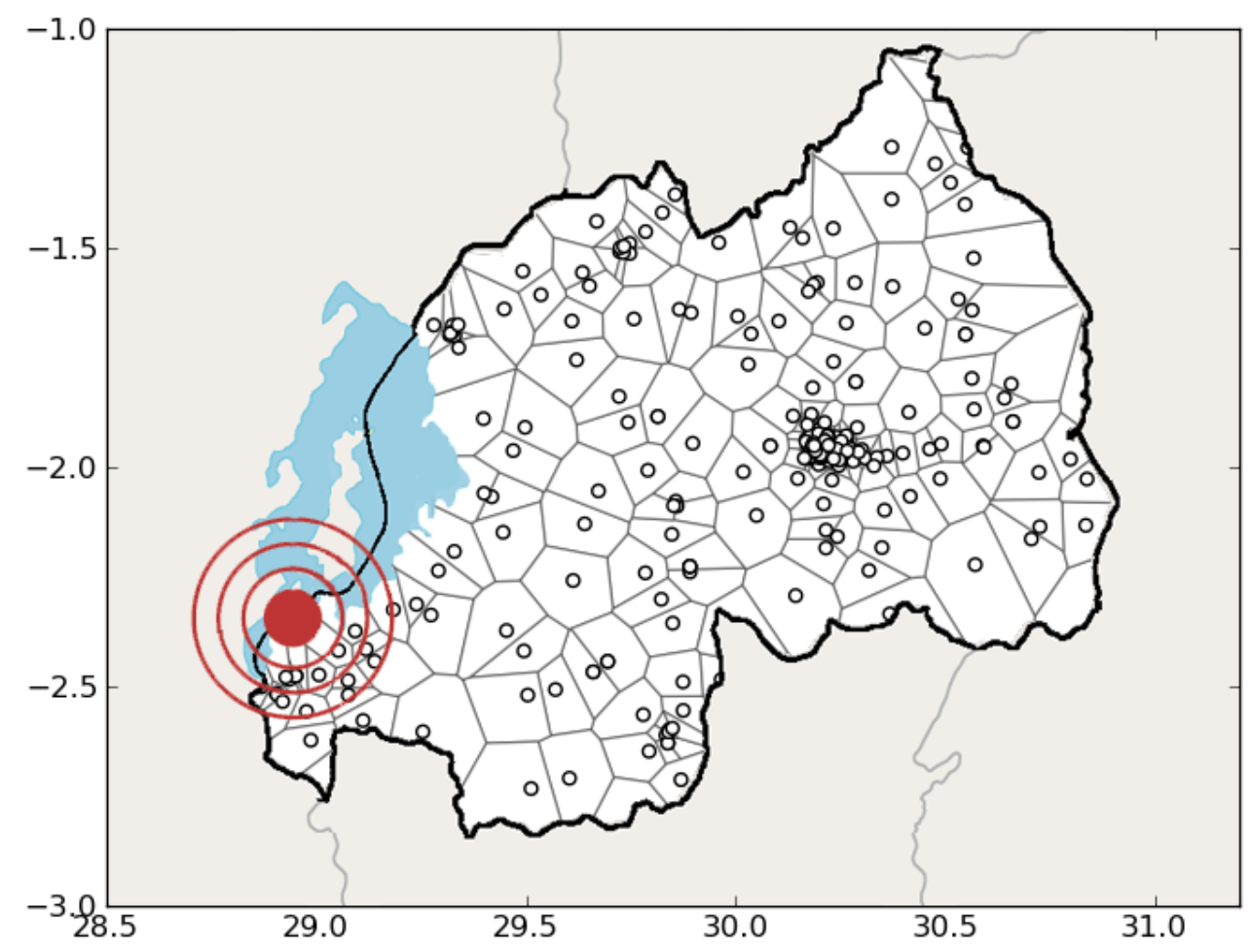

Figure 1: Map of Rwanda showing the location of mobile phone towers (as of February 2008) and the location of the Lake Kivu earthquake of 2008. Each black dot represents a cell tower, with the approximate area covered by the tower demarcated by adjacent Voronoi cells. The epicenter of the earthquake is shown with red concentric circles. 


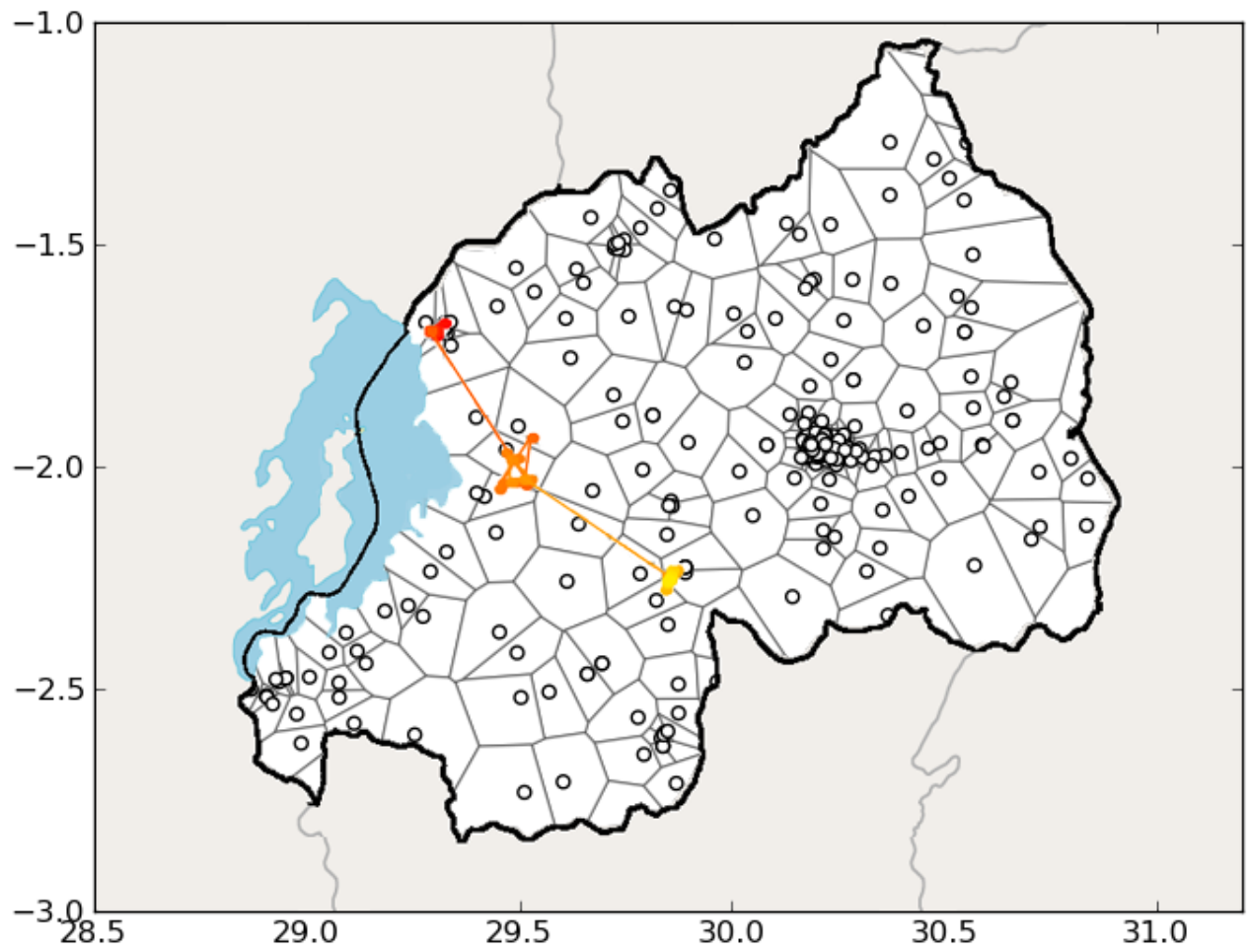

Figure 2: Map of Rwanda showing a single individual's inferred trajectory over a 6 month period. Although the individual only makes a small number of phone calls, the locational inference algorithm is able to roughly assign the user a continuous trajectory through time to places not restricted to the set of known locations of cellular towers. This particular individual is observed to slowly migrate southeastward, with early locations colored dark red and later locations colored yellow. 


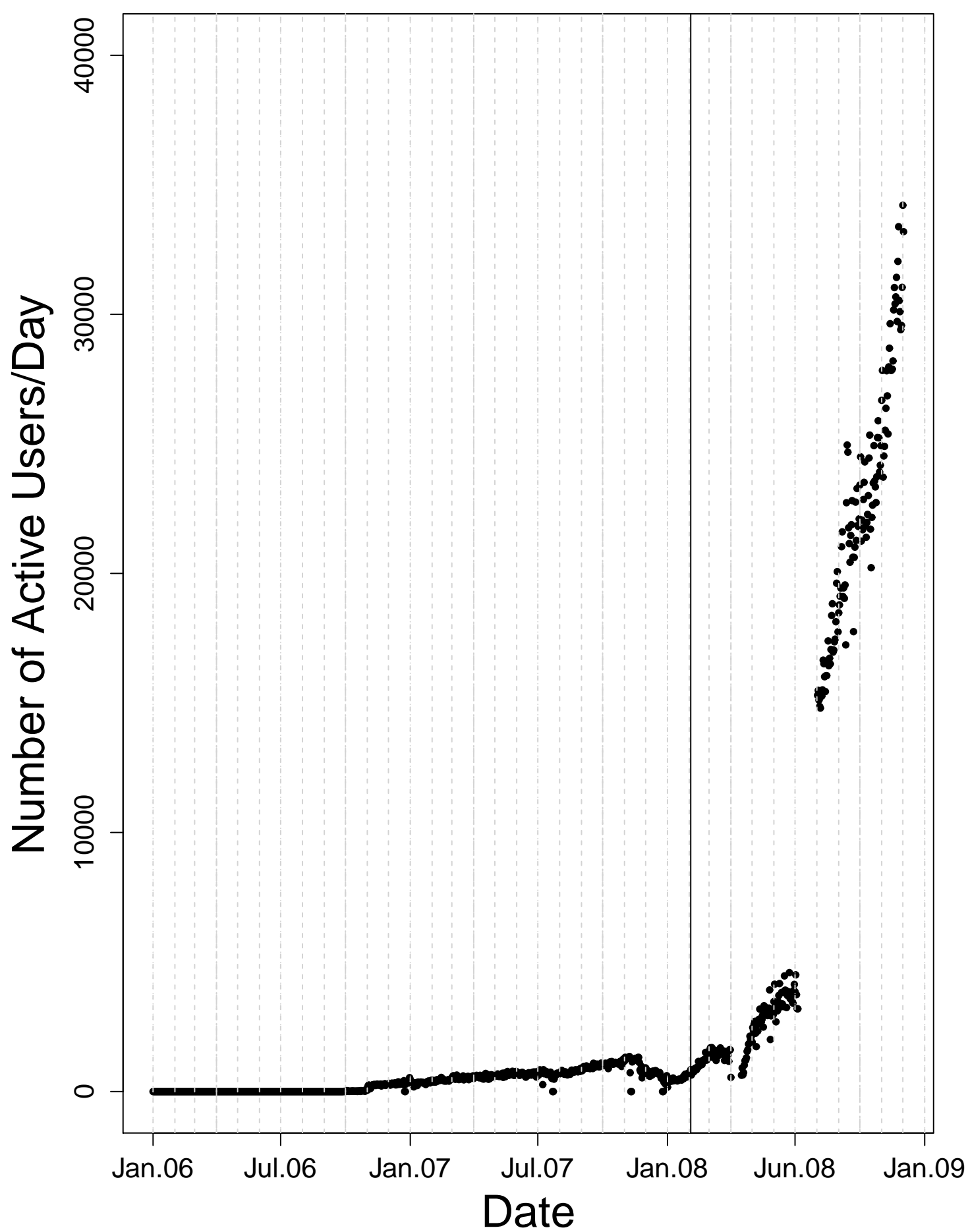

Figure 3: Growth in active users of the Mobile Money transfer system, using data from January 2006 until January 2009. The service was first launched in late 2006, and uptake was low at the time of the Lake Kivu earthquake, which is represented by the vertical line on February 3, 2008. Use of the service increased rapidly beginning in mid-2008, when the operator began heavily advertising and promoting the product. Gaps indicate days with missing data (most notably from June 2008). 


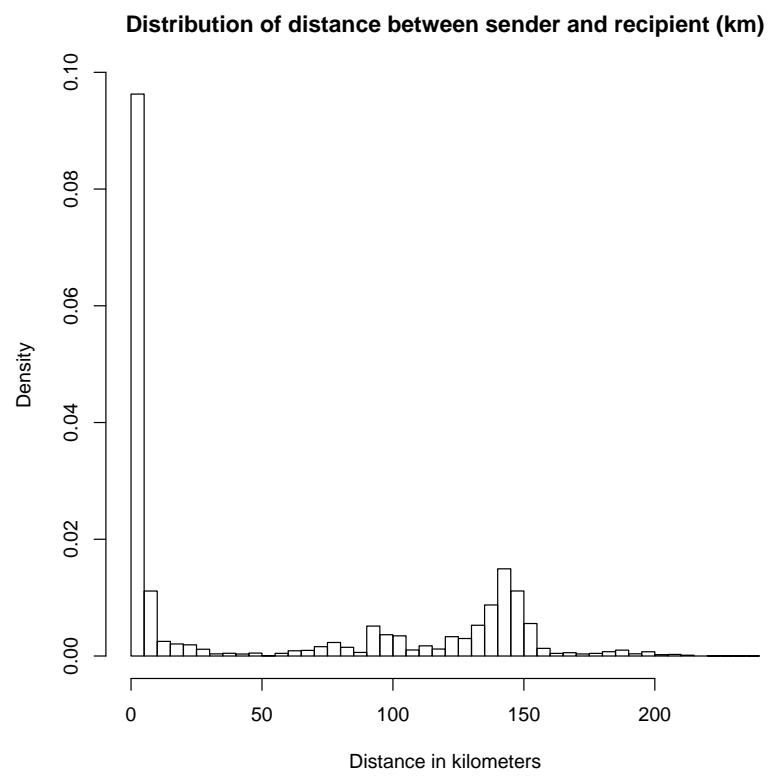

(a) Transfer distances over 4 years

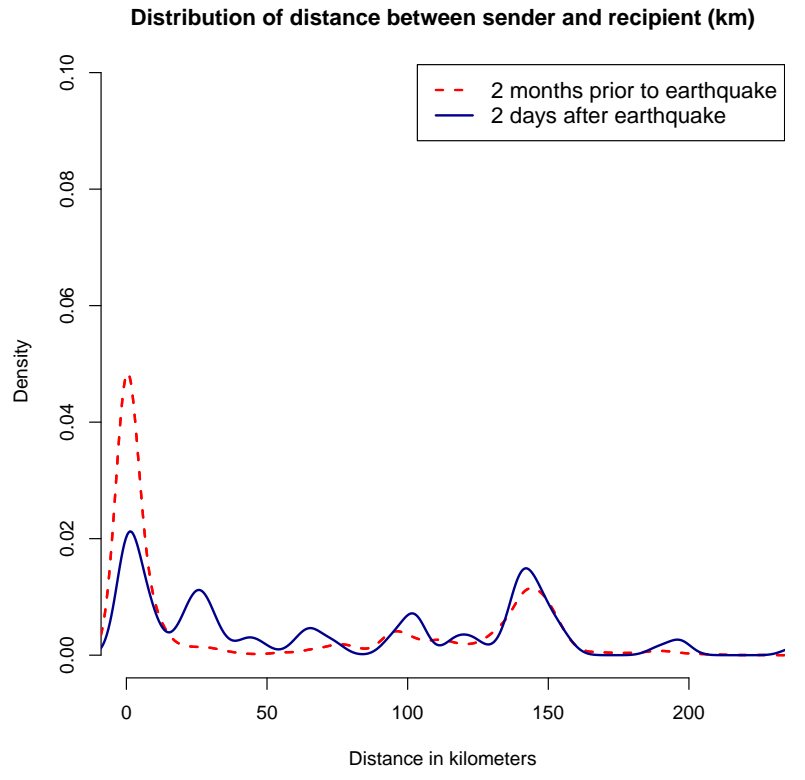

(b) Transfer distances just before/after quake

Figure 4: Distribution of distances over which transfers are sent to and from the earthquake region. A Kolmogorov-Smirnow test rejects the equality of the two distributions in (b) with $p \leq 0.001$. 


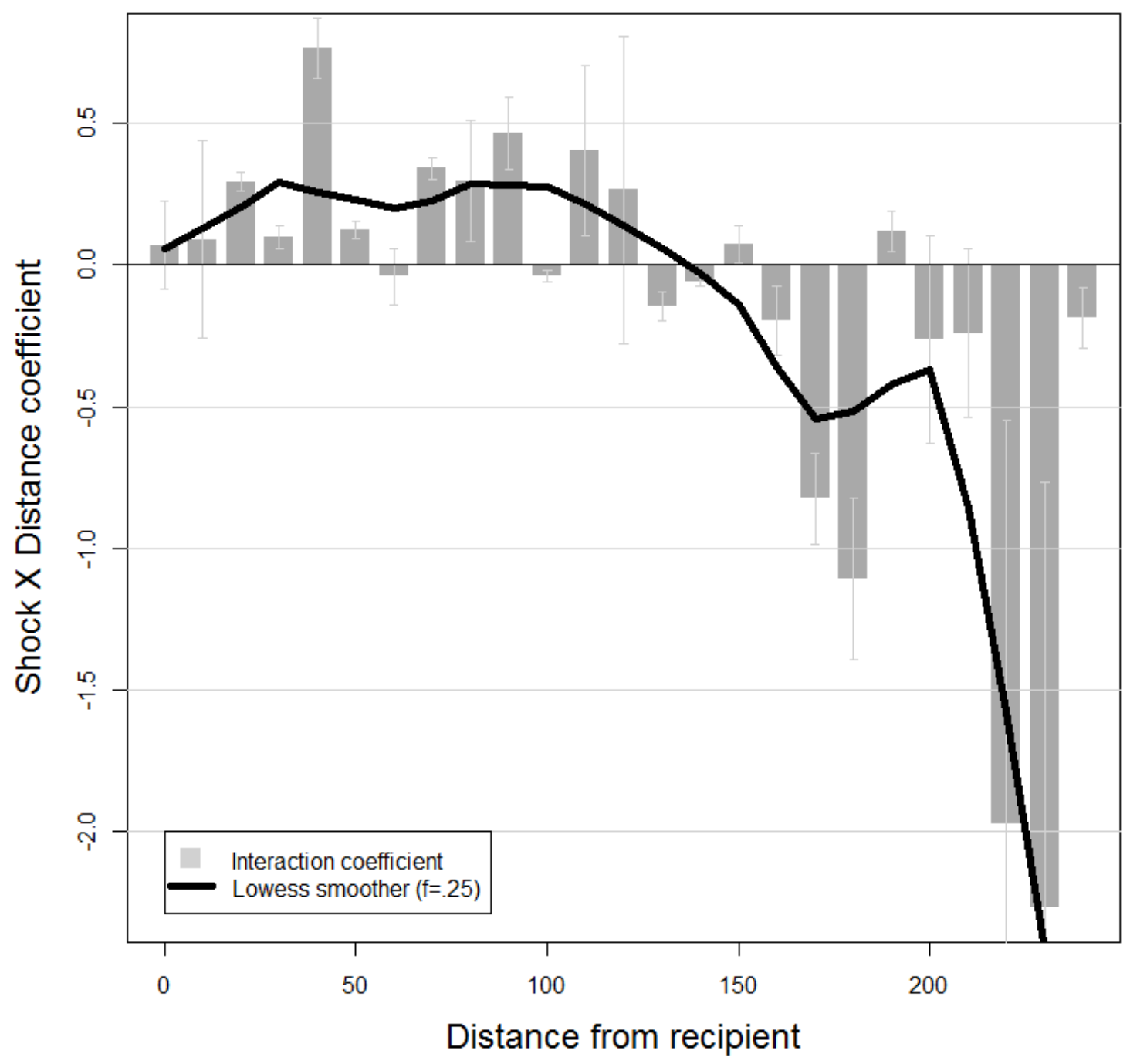

Figure 5: Relationship between the geographic structure of an individual's network and her propensity to receive a transfer after the earthquake. Values on the y-axis correspond to the estimated coefficients from on $\mu_{2}$ from regression (5), where $Z_{i}$ is the number of $i$ 's contacts who live within $R$ kilometers of $i$, with $R$ plotted on the x-axis. 


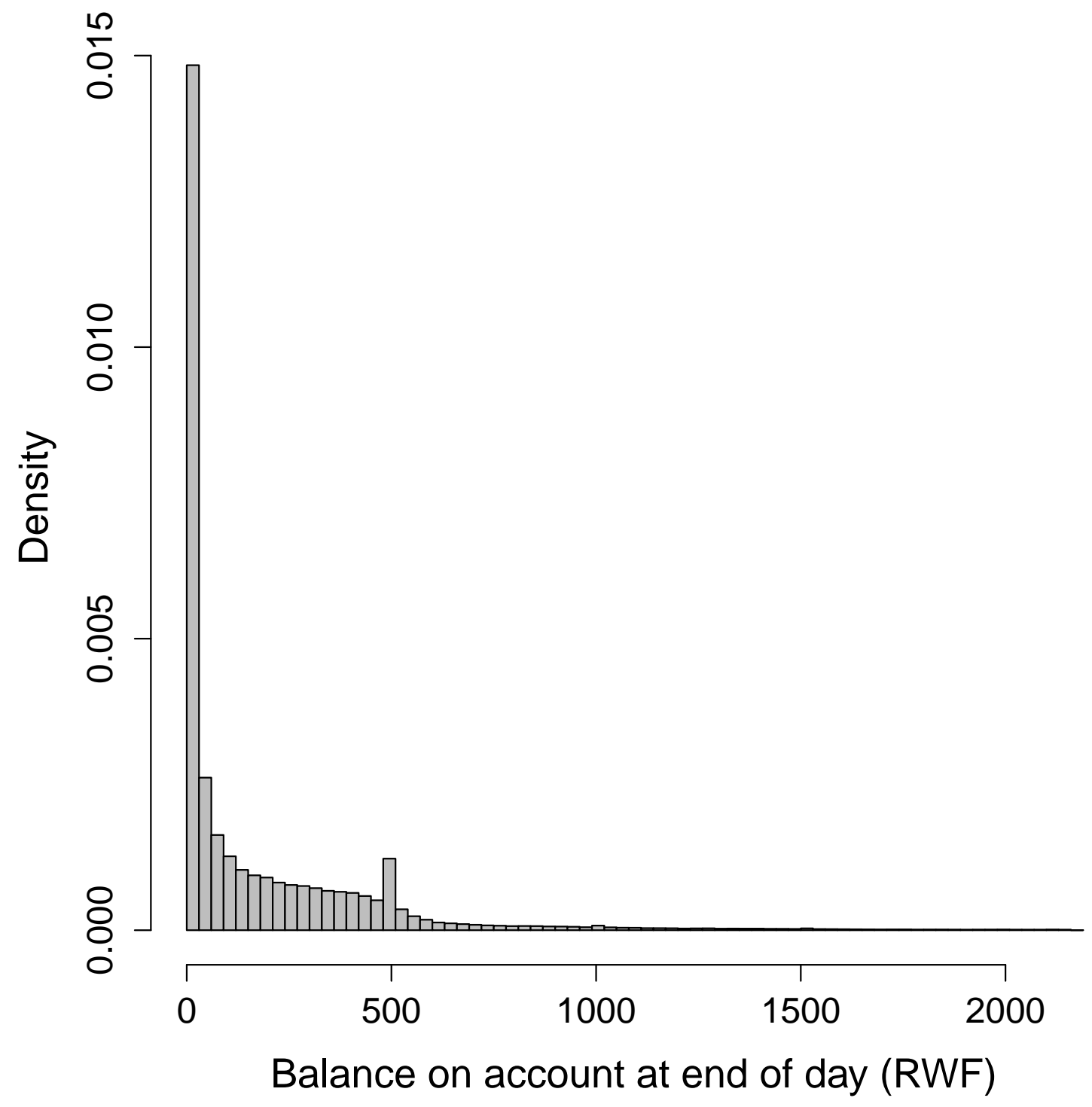

Figure 6: Distribution of end-of-day account balances on February 1, 2008, the day before the Lake Kivu earthquake. Subscribers with balances in the top and bottom 0.5 percent of all users were removed to improve the quality of the display (one user had a balance of 442500RWF). 


\section{Appendices}

\section{A Motives for Mobile Phone-Based Giving: Details}

\section{A.1 Charity}

As discussed in the main text, the mobile phone-based giving that occurs in response to economic shocks is statistically strong, though small in absolute terms. We defined a simple model of charity, by which we refer to the broad class of motives where a giver gives because he receives direct utility from the act of giving or from increasing the utility of another. The canonical example of this behavior is pure altruism, where one person's utility depends positively on another's (Becker 1976, Andreoni \& Miller 2002):

$$
U_{i t}=u_{i}\left(x_{i t}-\tau_{j i t}\right)+\gamma_{i j} u_{j}\left(x_{j}+\tau_{j i t}\right)
$$

As before, we denote by $\tau_{j i t}$ a transfer sent from $i$ to $j$ at time $t$. Assuming $u_{i}(\cdot)$ and $u_{j}(\cdot)$ are increasing and concave, with $x_{i}$ representing the income of individual $i$ and $\gamma_{i j}$ denoting the level of altruism felt by $i$ toward $j$, it is easily shown that two predictions of such a model are

$$
\frac{\partial E\left[\tau_{j i} \mid x_{i}\right]}{\partial x_{i}} \geq 0 \quad \text { and } \quad \frac{\partial E\left[\tau_{j i} \mid x_{j}\right]}{\partial x_{j}} \leq 0
$$

Giving is expected to increase in the income of the sender (as the marginal cost of giving decreases), and decrease in the income of the recipient (as the marginal benefit of a gift decreases). Such predictions are supported by observed patterns of altruism in a variety of contexts, including charitable giving in the United States (Andreoni 2006) and the behavior of "rescuers" in Nazi-occupied Europe during World War II (Hoffman 2010). ${ }^{20}$

Through similar logic, we expect that in cases of directed altruism, where $\gamma_{i j}$ varies across dyads, transfers decrease with the social distance between $i$ and $j$. However, ceteris paribus and conditional on an appropriate measure of social distance, there should be no relationship between transfers and geographic distance. Finally, in the simple model specified by (8), we expect current transfers between $i$ and $j$ to be "memory-less" (Fafchamps \& Lund 2003), i.e. current transfers should not depend on the past history of $i$ - $j$ transfers so as long as we can control for other time-invariant aspects of the $i$ - $j$ relationship. To the extent that an

\footnotetext{
${ }^{20}$ While the comparative statics in (A.2) are most transparent in the model of linear altruism specified by equation (8), similar predictions obtain from several related models of charitable behavior, and we make no pretense to be able to distinguish between them. Thus, models of inequity and inequality aversion (where $i$ seeks to minimize $\left|x_{i}-x_{j}\right|$ ), social welfare models (where $i$ maximizes $\min \left\{x_{1}, \ldots, x_{n}\right\}$ ), and warm glow giving (where $v\left(x_{j}+\tau_{j i}\right)$ in (8) is replaced with $\tau_{j i}$ ) all yield similar predictions (cf. Fehr \& Schmidt 1999, Bolton \& Ockenfels 2000, Charness \& Rabin 2002, Andreoni 1990, List \& Lucking-Reiley 2002).
} 
association does exist between past transfers and current transfers, we would expect it to be positive, as past transfers may reveal information about how much $i$ cares about $j$, above and beyond the undirected measures of relationship strength that we employ. These predictions are summarized in column (3) of Table 8.

\section{A.2 Conditional reciprocity}

By conditional reciprocity, we refer to motives that are embedded in long-term relationships of bilateral exchange. We focus here on instrumental reciprocity, where mutual exchange is motivated by the expectation of future reciprocation (cf. Coate \& Ravallion 1993, Karlan et al. 2009), as this model most transparently leads to empirical predictions that can be tested with the data at our disposal. Other models of reciprocity, and most notably the intrinsic, preference-based reciprocity modeled by Rabin (1993) and Falk \& Fischbacher (2006), produce similar predictions. However, since our intent is not to differentiate between these different types of conditional reciprocity, we present a simple model of dynamic limited commitment that captures many of the central tenets of the wider literature. ${ }^{21}$

The basic idea is easily illustrated by a model of risk sharing under dynamic limited commitment (Ligon et al. 2002, Foster \& Rosenzweig 2001). Following Foster \& Rosenzweig (2001), we assume $i$ has stationary, single-period utility specified by (8), but allow for the possibility that $i$ expects to benefit from future interaction with $j$ :

$$
U_{i t}=u_{i}\left(x_{i t}-\tau_{j i t}\right)+\gamma_{i j} u_{j}\left(x_{j t}+\tau_{j i t}\right)+E \sum_{s=t+1}^{\infty} \delta^{s-t}\left[u_{i}\left(x_{i s}-\tau_{j i s}\right)+\gamma_{i j} u_{j}\left(x_{j s}+\tau_{j i s}\right)\right]
$$

The first part of this equation is identical to the altruistic model (8), while the second term captures the discounted expected utility of the relationship.

This formulation produces two key insights relevant to the current analysis. First, when contracts are not fully enforceable ex-post, transfers received in the current period will depend on past transfers given. Models using conditional reciprocity norms generate the same prediction. For the LC model, this property is formally derived in Appendix A.3, however the intuition is straightforward: In the stationary model of (8), $i$ and $j$ will transfer the necessary $\tau_{j i t}$ to equate the ratio of their ex post marginal utilities to $\gamma$ (or $1 / \gamma$ if $x_{i}<x_{j}$ ). If $\gamma$ is sufficiently small, $i$ and $j$ operate in autarky. By contrast, in the dynamic model specified by (9), $i$ and $j$ also derive utility from expected future interactions and transfers, and so may adjust the ratio of marginal utilities at time $t$ to maintain the relationship and avoid reversion to a series of static Nash

\footnotetext{
${ }^{21}$ For recent experimental work that differentiates between different types of reciprocity, see Leider et al. (2009), Ligon \& Schechter (2011), and Cabral et al. (2011). Kinnan (2014) provides a test between different barriers to informal insurance in rural villages.
} 
equilibria. Intuitively, when $i$ suffers a shock in period $t$, the marginal utility of $\tau_{i j t}$ will be quite high and so he will be willing to sacrifice a greater share of his continuation utility in exchange for a larger transfer at $t$. As a result, we expect transfers from $i$ to $j$ sent in response to a shock to be decreasing in the net balance of transfers previously made from $i$ to $j$ (denoted by $T_{j i t}^{\text {net }}$ ):

$$
\frac{\partial E\left[\tau_{j i t} \mid T_{j i t}^{n e t}\right]}{\partial T_{j i t}^{n e t}} \leq 0 \quad \text { where } \quad T_{j i t}^{n e t}=\sum_{s=0}^{t-1} \tau_{j i s}-\tau_{i j s}
$$

As robustness checks, we further expect that $\tau_{j i t}$ will decrease in the gross volume of prior transfers from $i$ to $j$ (denoted by $T_{j i t}$ ) and increase in gross prior transfers from $j$ to $i$ (denoted by $T_{i j t}$ ):

$$
\frac{\partial E\left[\tau_{j i t} \mid T_{j i t}\right]}{\partial T_{j i t}} \leq 0 \quad \text { and } \quad \frac{\partial E\left[\tau_{j i t} \mid T_{i j t}\right]}{\partial T_{i j t}} \geq 0 \quad \text { where } \quad T_{j i t}=\sum_{s=0}^{t-1} \tau_{j i s}
$$

This model of dynamic limited commitment has been shown to account for empirical evidence better than a stationary model with no history-dependence (Ligon et al. 2002, Genicot \& Ray 2003, Fafchamps \& Lund 2003).

A second point of contrast between the model of charity (8) and conditional reciprocity (9) pertains to the marginal effect of geographic distance on transfers. Under the second formulation, and as discussed by Ligon (1998) and De Vreyer et al. (2010), transfers are expected to decrease as, ceteris paribus, the cost of monitoring and enforcement increases. Assuming that such monitoring and enforcement costs increase monotonically with geographic distance $D_{i j}$, we expect that: ${ }^{22}$

$$
\frac{\partial E\left[\tau_{i j} \mid d_{i j}, S_{i j}\right]}{\partial d_{i j}} \leq 0
$$

i.e., conditional on the strength of the social connection between $i$ and $j$, the further away $j$ resides from $i$, the less likely is $j$ to help $i$ following an idiosyncratic shock. Of course, distance is likely correlated with other factors that influence the decision to give, but which are not related to monitoring and enforcement per se. ${ }^{23}$ Thus, when we operationalize (A.5) in a regression setting, we always condition our empirical results on $S_{i j}$, an undirected measure of the strength of the relationship between $i$ and $j$, and a dyad fixed effect $\pi_{i j}$, so that we estimate the partial effect of geographic distance holding constant other unobservable characteristics of the dyad.

The predictions of model (9) are summarized in column (4) of Table 8. Unlike the model of charity, the

\footnotetext{
${ }^{22}$ This could be the case if, for instance, $j$ must visit $i$ to verify damage to $i$ 's property, and the cost of travel increases with distance.

${ }^{23}$ For instance, transaction costs, which often rise with distance, have received recent attention by Jack \& Suri (2012). However, since the cost of remitting over the Rwandan mobile network is free at all distances, transaction costs are unlikely to drive empirical estimates of (A.5).
} 
model of reciprocity does not make strong predictions regarding the relative wealth of $i$ and $j$. We might expect transfers to go to wealthier individuals if strategic agents seek to ingratiate themselves for future reciprocation. Such an interpretation is supported by recent work by Schechter \& Yuskavage (2011), who find that transfers are likely to flow from more to less wealthy households in unreciprocated relationships, while reciprocated relationships are more likely between wealthier households. Alternatively, we could observe flows from the rich to the poor if the poor reciprocate in ways other than airtime (Fafchamps 1999, Platteau 1995).

In Section 5, we will estimate the partials of equations (4) and (5) to adjudicate between the conflicting predictions of models (8) and (9). First, however, we describe the data used in the analysis, as well as our methods for measuring each component of our empirical specifications.

\section{A.3 History-Dependence in Reciprocity}

We derive the history-dependence of current period transfers in the model of dynamic limited commitment presented in Section A.2. The exposition is based on Ligon et al. (2002) and Foster \& Rosenzweig (2001), but makes explicit the extension to the case where single-period utility includes a component of altruism. Other models of reciprocity, and in particular the preference-based reciprocity of Rabin (1993) and Falk \& Fischbacher (2006), are predicated on fundamentally different "reciprocal" motives, but yield similar empirical predictions. We employ the enforced/instrumental model because the comparative statics that result are most directly testable with the data at our disposal, but do not mean to imply that the behavior we observe is necessarily motivated by this particular type of reciprocity vs. a different type of reciprocity.

We rely on a model with two agents $i$ and $j$ with von Neumann-Morgenstern utility where $i$ 's single period utility in increasing in $j$ 's single period utility according to (8), and vice versa. In static equilibrium, non-zero transfers occur when $i$ 's marginal utility of consumption is less than the $\gamma$-weighted utility of $j$, i.e., when

$$
u_{i}^{\prime}\left(x_{i t}\right)<\gamma u_{j}^{\prime}\left(x_{j t}\right)
$$

or when the converse applies to $j .{ }^{24}$ Call this static transfer, which depends on the state $s$ of the world, $\tau_{j i t}^{N}$, where the superscript $N$ denotes that this is the static Nash equilibrium. It is easily seen that whenever (A.6) holds, $i$ will transfer a non-zero $\tau_{j i t}^{N}$ that satisfies

$$
\frac{u_{i}^{\prime}\left(x_{i t}-\tau_{j i t}^{N}\right)}{u_{j}^{\prime}\left(x_{j t}+\tau_{j i t}^{N}\right)}=\gamma
$$

When the converse of (A.6) applies (i.e. $u_{j}^{\prime}\left(x_{j t} \leq \gamma u_{i}^{\prime}\left(x_{i t}\right)\right)$, the transfer will be negative, and in all other

\footnotetext{
${ }^{24}$ For simplicity we assume that $i$ and $j$ are similarly altruistic, i.e., $\gamma_{i j}=\gamma_{j i}=\gamma$.
} 
cases the transfer will be zero.

In the repeated-game setting, agents are infinitely lived but are unable to save across periods. Given uncertainty as to the state of the world that will be realized in the future, both $i$ and $j$ can potentially be made better off through (possibly negative) state-contingent transfers $\tau_{j i t}$. The current-period utility of $i$ is then the sum of single period utility plus the expected discounted utility of future interaction with $j$, where $\delta$ is the discount factor:

$$
U_{i t}^{T}=\underbrace{\underbrace{u_{i}\left(x_{i t}-\tau_{j i t}\right)}_{\text {own consumption }}+\underbrace{\gamma u_{j}\left(x_{j t}+\tau_{j i t}\right)}_{\text {altruistic benefit }}}_{\text {single period utility }}+\underbrace{E \sum_{s=t+1}^{\infty} \delta^{s-t}\left[u_{i}\left(x_{i s}-\tau_{j i s}\right)+\gamma u_{j}\left(x_{j s}+\tau_{j i s}\right)\right]}_{\text {continuation value of relationship }}
$$

Though $i$ and $j$ may agree ex ante to a set of state-contingent transfers, imperfect ability to enforce contracts implies limited commitment ex post. After the state of the world is realized, either agent can renege at any time, in which case both $i$ and $j$ will revert to the static Nash equilibrium where $\tau_{j i t}=\tau_{j i t}^{N}$ as given by (A.7). Then, $i$ 's utility is simply

$$
U_{i t}^{A}=u_{i}\left(x_{i t}-\tau_{j i t}^{N}\right)+\gamma u_{j}\left(x_{j t}+\tau_{j i t}^{N}\right)+E \sum_{s=t+1}^{\infty} \delta^{s-t}\left[u_{i}\left(x_{i s}-\tau_{j i s}^{N}\right)+\gamma u_{j}\left(x_{j s}+\tau_{j i s}^{N}\right)\right]
$$

The set of sustainable contracts are then defined by the implementability constraints that require utility for both agents to be (weakly) greater than under the static Nash equilibrium, i.e., $U_{i t}^{T} \geq U_{i t}^{A}$ and $U_{j t}^{T} \geq U_{j t}^{A}$. These constraints specify a superset of sustainable contracts, a subset of which are efficient.

In the repeated game, the transition matrix $\Pi$ specifies the probabilities $p_{s r}=\operatorname{Pr}\left(X_{t+1}=r \mid X_{t}=s\right)$ of transitioning from state $s$ at time $t$ to state $r$ at time $t+1$, where $S$ is the state space of the Markov chain. The set of constrained-efficient contracts along the Pareto frontier can then be characterized as

$$
U_{j s}\left(U_{i s}\right)=\max _{\tau_{j i t}}\left\{u_{j}\left(x_{j t}+\tau_{j i t}\right)+\gamma u_{i}\left(x_{i t}-\tau_{j i t}\right)+\delta \sum_{r \in S} p_{s r} U_{j r}\left(U_{i r}\right)\right\}
$$

Where $U_{i s}$ denotes the expected discounted utility of $i$ given state $s$ at time $t$, which $j$ is required to satisfy. 
The following conditions must be met for (A.10) to be optimal:

$$
\begin{aligned}
\lambda: & {\left[u_{i}\left(x_{i t}-\tau_{j i t}\right)-u_{i}\left(x_{i t}-\tau_{j i t}^{N}\right)\right]+\gamma\left[u_{j}\left(x_{j t}+\tau_{j i t}\right)-u_{j}\left(x_{j t}+\tau_{j i t}^{N}\right)\right]+\delta \sum_{r \in S} p_{s r} U_{i r} \geq 0 } \\
\delta p_{s r} \phi_{r}: & U_{i r} \geq 0 \\
\delta p_{s r} \mu_{r}: & U_{j r}\left(U_{i r}\right) \geq 0 \\
\psi_{i}: & x_{i t}-\tau_{j i t} \geq 0 \\
\psi_{j}: & x_{j t}+\tau_{j i t} \geq 0
\end{aligned}
$$

With $u_{i}(\cdot)$ and $u_{j}(\cdot)$ concave, $U_{i}(\cdot)$ and $U_{j}(\cdot)$ are also concave, implying the following first order conditions (??-??) and envelope condition (A.11):

$$
\begin{aligned}
\frac{u_{j}^{\prime}\left(x_{j t}+\tau_{j i t}\right)+\gamma u_{i}^{\prime}\left(x_{i t}-\tau_{j i t}\right)}{u_{i}\left(x_{i t}-\tau_{j i t}+\gamma u_{j}^{\prime}\left(x_{j t}+\tau_{j i t}\right)\right.} & =\lambda+\frac{\psi_{i}-\psi_{j}}{u^{\prime}\left(x_{i t}-\tau_{j i t}\right)+\gamma v^{\prime}\left(x_{j t}+\tau_{j i t}\right)} \\
-V_{r}^{\prime}\left(U_{r}\right) & =\frac{\lambda+\phi_{r}}{1+\mu_{r}} \\
\lambda & =\frac{u_{j}^{\prime}\left(x_{j t}+\tau_{j i t}\right)+\gamma u_{i}^{\prime}\left(x_{i t}-\tau_{j i t}\right)}{u_{i}^{\prime}\left(x_{i t}-\tau_{j i t}\right)+\gamma u_{j}^{\prime}\left(x_{j t}+\tau_{j i t}\right)} \\
& =-V_{s}^{\prime}\left(U_{s}\right)
\end{aligned}
$$

The optimal contract is thus characterized by the slope of the Pareto frontier, $\lambda$, which determines the extent to which $i$ can transfer current-period utility to $j$. For each state, there exists a set of implementable points on the Pareto frontier between $\lambda_{s}^{\text {min }}$ and $\lambda_{s}^{\max }$.

The history dependence arises as follows. When $i$ and $j$ first enter a relationship at $t=0$, they agree upon a contract that specifies a fixed ratio of marginal utilities $\lambda_{0}$, which is a point on the Pareto frontier. Once the state of nature becomes known at $t=1, i$ and $j$ will attempt the $\tau_{j i t}^{*}$ that maintains the ratio of marginal utilities at $\lambda_{0}$. For simplicity, assume that at $t=1, j$ suffers a negative shock such that $x_{i 1}>x_{j 1}$, though the same logic applies when $x_{i 1} \leq x_{j 1}$. Then, in order to maintain $\lambda_{0}$, the agents will attempt $\tau_{j i t}^{*}>0$. However, if $\tau_{j i t}^{*}>0$ is large enough to make the implementability constraint on $i$ bind, then $i$ and $j$ will implement $\tau_{j i t}<\tau_{j i t}^{*}$ in order to avoid the static Nash equilibruim $\tau_{j i t}^{N}$ and preserve the continuation value of the relationship. The implemented $\tau_{j i t}$ will be just sufficient to relax the constraint on $i$, and a new constrained-efficient ratio of single-period marginal utilities $\lambda_{t}$ will be determined according to (??).

If the implementability constraints never bind (intuitively, this occurs when income covariance between $i$ and $j$ is high), then agents will continue to equate marginal utilities at $\lambda_{0}$ and there will effectively be no history dependence in $\tau_{j i t}$. However, when a constraint binds, the dynamic model allows the worse-off agent 
to sacrifice future consumption (by accepting a less favorable ratio of utility $\lambda$ ) in exchange for a transfer in the current period. This recalibration of $\lambda$ affects all subsequent $\tau_{j i t}$. 


\section{B Robustness}

\section{B.1 Functional form assumptions}

We briefly show that our central results are not sensitive to the precise econometric specification, or to the choice of time window (which in most regressions is restricted to the period starting one month before the earthquake and ending one month after the earthquake). Appendix Table 1 presents estimates of the average treatment effect of model (1) using the full dataset from October 2006 until July 2009 under a variety of econometric specifications. Column (1) gives the standard OLS results with no control variables $X_{r t}$, time fixed effects $\theta_{t}$, or tower fixed effects $\pi_{r}$. Column (2) includes time-varying controls to account for regional variation in mobile phone use, column (3) adds regional fixed effects, and column (4) adds daily dummy variables. Across all specifications, the estimated effect of the shock remains strong and significant, and of a magnitude similar to that presented in Table 3.

\section{B.2 Sensitivity to Outliers}

To address the possibility that our effects are driven in part by outliers or a dependent variable with a long right tail, we run a series of robustness checks in Appendix Table 2. Column (1) contains the original results from column (4) of Table 3 for reference. Column (2) transforms the dependent variable $\left(\tau_{i j t}\right)$ into a binary indicator that is one when $\tau_{i j t}>0$ and zero otherwise. Column (3) trims outliers by dropping observations with $\tau_{i j t}>2500 \mathrm{RWF}$, where 2500RWF is the 99th percentile of non-zero transfers received. Column (4) winsorizes the dependent variable at the 99th percentile following Tukey (1962) by replacing all values greater than the 99th percentile (2500RWF) with values at the 99th percentile. Column (5) applies the inverse hyperbolic sine transformation to reduce the influence of extreme observations of dependent variables (Burbidge et al. 1988). In all cases, the primary coefficient of interest is still positive and statistically significant.

\section{B.3 Robustness of Dyadic Regressions}

In the body of the paper, we use dyadic regressions to measure which types of individuals $i$ and $j$ are most likely to receive and send transfers in response to the earthquake. Tables 4 and 7 employ dyad-specific fixed effects to control for unobserved, time-invariant characteristics of the dyad. Thus, the coefficient on the interaction between $x_{i}$ and $S h o c k_{i t}$ in Table 4 indicates the extent to which wealthier individuals $i$ are more likely to receive a transfer after the earthquake, in relation to the normal activity observed between $i$ and $j$. This specification, formalized in equation (5), reduces biases resulting from unobserved characteristics of $i$, 
$j$, and the dyad $i$ - $j$ that could be correlated with the error term $\epsilon_{i j t}$. For instance, if $i$ sends a large transfer to $j$ on each day of the year (for reasons unrelated to economic shocks), the dyadic fixed effects will ensure that a large transfer sent from $j$ to $i$ on the day of the earthquake is not mis-attributed to the effect of the earthquake.

As a robustness check, we demonstrate that our key results hold if the regressions are estimated with a more parsimonious model that replaces the dyad-specific fixed effects (for each pair $i$-j) with sender-specific fixed effects (for each sender $j$ ). This model is slightly less restrictive, and more directly corresponds to the intuition that motivates the dyadic results, i.e., that we wish to identify the types of people $i$ (where type is proxied by $x_{i}$ ) are chosen by $j$ to receive a transfer, conditional on $j$ 's average behavior. Formally, we estimate the following model:

$$
\begin{aligned}
\tau_{i j t}= & \delta_{0}+\delta_{1} \text { Shock }_{i t}+\delta_{2} x_{i}+\delta_{3} x_{i} \text { Shock }_{i t}+\delta_{4} x_{j} \text { Shock }_{i t}+ \\
& \delta_{5} \text { NearEpicenter }_{i t}+\delta_{6} x_{i} \text { NearEpicenter }_{i t}+\delta_{7} x_{j} \text { NearEpicenter }_{i t}+ \\
& \delta_{8} x_{i} \text { DayOfShock }_{t}+\delta_{9} x_{j} \text { DayOfShock }_{t}+\theta_{t}+\pi_{j}+\varepsilon_{i j t}
\end{aligned}
$$

While the full specification of (B.1) is somewhat dense, all of the coefficients of interest are contained on the first line, represented by $\delta_{1}-\delta_{4}$. The remaining $\delta_{5}-\delta_{9}$ are sub-interaction terms that are included for consistency but which have limited real-world significance. To interpret, $\delta_{1}$ indicates whether individuals affected by the earthquake (for whom NearEpicenter ${ }_{i t}=1$ and DayOfShock D $_{t}=1$ ) are more likely to receive a transfers; $\delta_{2}$ indicates whether wealthier individuals are more likely to receive more under normal circumstances; $\delta_{3}$ indicates whether wealthier individuals receive more because of the earthquake; and $\delta_{4}$ indicates whether wealthier individuals send more to friends affected by the earthquake. All estimates are conditional on the average amount sent by $j$.

Results from estimating model (B.1) are presented in Appendix Table 4. Only very minor differences exist between these results and those presented in Table 4. Transfers sent in response to the earthquake increase significantly in the wealth of the recipient, but are not significantly related to the wealth of the sender. In other words, holding the identity of the sender fixed, it is the wealthier individuals - not the poorer individuals predicted by models of charity - who are most likely to receive a transfer.

\section{B.4 Measuring social proximity}

In the regression results presented in the body, we control for the strength of the relationship between $i$ and $j$ with $S_{i j}$, which is simply the total number of calls made between $i$ and $j$ (in either direction) in the year 
prior to the window of time used in the regressions. We choose this metric as a simple and easy to compute statistic that is likely to be correlated with the overall social proximity of $i$ and $j$. However, several other such measures of $S_{i j}$ are also reasonable. In particular, Karlan et al. (2009) and Leider et al. (2009) suggest a related metric, network flow, which captures the number of distinct paths between $i$ and $j$ through third parties $k$. The intuition is that each common friend $k$ increases the shared social collateral between $i$ and $j$.

In Appendix Table 5, we show that our results are not sensitive to the specific measure of social proximity used. The estimates in Appendix Table 5, which utilize network flow to measure $S_{i j}$, are quite similar to the estimates in Table 4, which measure $S_{i j}$ as the total number of prior phone calls. Controlling for network flow in the other regressions similarly has no effect.

\section{B.5 Standard errors}

As discussed in Section 3, for standard error estimates to be consistent in the dyadic regressions, they should ideally be cross-clustered by sender $i$ and recipient $j$. This is because transfers involving the same individual are likely to be correlated with each other - e.g., if $j$ transfer airtime to $i$, he is ceteris paribus less able to transfer airtime to others. In the results presented so far we have clustered standard errors by the district in which the recipient resides.

As a robustness check, Appendix Table 3 compares alternative methods of obtaining standard errors using different levels of clustering: no clustering (column 1), by recipient (column 2), by sender (column 3), and by date (column 4). Standard errors are largest when we cluster by recipient, but in all specifications the coefficients of interest remain significant. 